\title{
On the four-point function of the stress-energy tensors in a CFT
}

\section{Anatoly Dymarsky}

Skolkovo Institute of Science and Technology, Skolkovo Innovation Center, Nobel street 3, Moscow, 143026, Russia

E-mail: dymarsky@skoltech.ru

ABSTRACT: We discuss to what extent the full set of Ward Identities constrain the fourpoint function of the stress-energy tensors or conserved currents in a conformal field theory. We calculate the number of kinematically unrestricted functional degrees of freedom governing the corresponding correlators and find that it matches the number of functional degrees of freedom governing scattering amplitudes of some "dual" massless particles in the auxiliary Minkowski space. We also formulate the conformal bootstrap constraints for the correlators in question in terms of only unrestricted degrees of freedom. As a byproduct we find interesting parallels between solving Ward Identities in coordinate and momentum space.

Keywords: Conformal and W Symmetry, Conformal Field Models in String Theory

ARXIV EPRINT: 1311.4546 


\section{Contents}

1 Introduction 1

2 Imposing conservation in the coordinate space 3

2.1 Conservation constraints for conserved currents 4

2.2 Permutation symmetry I 4

2.3 Number of unrestricted functional degrees of freedom 5

2.4 Permutation symmetry II 6

2.5 Conformal bootstrap for unrestricted D.O.F. 8

$\begin{array}{lll}2.6 & \text { Small d and degenerate tensors } & 10\end{array}$

$\begin{array}{lll}2.7 & \text { Four point function of the stress-energy tensors } & 11\end{array}$

3 CFT correlators and scattering amplitudes 12

4 Two easy ways to solve the conservation constraints $\quad 14$

4.1 Solving all W.I.'s automatically 14

$\begin{array}{ll}4.2 \text { Solving Ward Identities in the momentum space } & 16\end{array}$

$\begin{array}{llr}5 & \text { Conclusions } & 20\end{array}$

$\begin{array}{ll}\text { A Degenerate tensors in various dimensions } & 20\end{array}$

B Conformal block decomposition in $\mathrm{d}=3 \quad 23$

\section{Introduction}

Conformal symmetry imposes powerful constraints on the correlation functions of primary operators in a conformal field theory. In particular, all two and three-point functions are fixed up to a small number of normalization constants [1-4]. At the same time the form of the four-point functions is not universal and depends on functions which encode the CFT's dynamics. In case of four scalars there is just one such function of the cross-ratios $u, v$

$$
\begin{aligned}
\left\langle\mathcal{O}\left(x_{1}\right) \mathcal{O}\left(x_{2}\right) \mathcal{O}\left(x_{3}\right) \mathcal{O}\left(x_{4}\right)\right\rangle & =\frac{f(u, v)}{\left(x_{1}-x_{2}\right)^{2 \Delta}\left(x_{3}-x_{4}\right)^{2 \Delta}}, \\
u=\frac{\left(x_{1}-x_{2}\right)^{2}\left(x_{3}-x_{4}\right)^{2}}{\left(x_{1}-x_{3}\right)^{2}\left(x_{2}-x_{4}\right)^{2}}, \quad v & =\frac{\left(x_{1}-x_{4}\right)^{2}\left(x_{2}-x_{3}\right)^{2}}{\left(x_{1}-x_{3}\right)^{2}\left(x_{2}-x_{4}\right)^{2}} .
\end{aligned}
$$

When the operators have spin $\mathcal{O}_{\mu \ldots}$ the four-point correlator depends on many such functions $f^{I}(u, v)$. 
In a general case the expression (1.1), or its generalization when the operators have spin, solves all Ward Identities ${ }^{1}$ with arbitrary $f^{I}(u, v)$. But in a special case when the operator dimension $\Delta$ saturates the unitary bound the operator $\mathcal{O}_{\mu \ldots}$ becomes conserved

$$
\partial_{\mu} \mathcal{O}_{\mu \ldots}=0
$$

In this case there are additional Ward identities that require that $\partial_{\mu} \mathcal{O}_{\mu \ldots}$ inside any correlator vanishes. At the level of two-point function this condition follows from conformal algebra and is automatically satisfied. At the level of three-point function conservation provides a set of linear constraints on the normalization coefficients. In the case of four-point function conservation of $\mathcal{O}_{\mu \ldots}$ yields a set of first order differential equations on $f^{I}(u, v)$ which further restrict possible form of the correlation function in question.

In this paper we analyze this system of coupled equations and calculate the number of unconstrained functional degrees of freedom governing the corresponding correlators. We were not able to solve these constraints explicitly. But we found that the number of the unconstrained degrees of freedom for the four-point function of the operators of spin $\ell_{i}$ in $\mathrm{d}$ dimensions matches precisely the number of functions $\mathrm{f}^{\prime}(s, t)$ of the Mandelstam variables governing most general scattering amplitude of four particles of spin $\ell_{i}$ in the $(d+1)$ dimensional Minkowski space. Thus our findings support and generalize an interesting connection between the CFT correlators and scattering amplitudes first observed in [5] and further generalized in $[6] .^{2}$

Our motivation to identify the degrees of freedom unconstrained by the Ward Identities is rooted, besides the usual aspiration to solve all available kinematic constraints explicitly, in the desire to apply the conformal bootstrap approach to the correlators with spin. The conformal bootstrap has proven to be a powerful tool to constrain CFT dynamics in various dimensions [9-21]. Yet to this moment the applications were limited to the four-point functions of the identical scalar operators. Certainly, considering operators with spin should yield more information, but technically it is much more difficult as the number of cumbersome constraints grows rapidly with spin. A particularly interesting case would be to consider the four-point function of the stress-energy tensors because the stressenergy tensor is the most universal operator present in all CFTs. Hence one might expect the corresponding constraints to be most fundamental. Although the resulting number of constraints is large (in a general case there will be 633 coupled equations), due to conservation $\partial_{\mu} T_{\mu \nu}=0$ many of them are not independent. If one succeeds to reformulate these constraints in terms of only unrestricted degrees of freedom the number of equations would reduce drastically (e.g. in $\mathrm{d}=3$ there will be just 5 such equations). To develop such a formalism is one of the goals of this paper.

This paper is organized as follows. In the next section we discuss general properties of the system of equations which encode conservation of operators at the level of correlation function. In particular we find the number of unrestricted degrees of freedom governing

\footnotetext{
${ }^{1}$ Namely, Poincaré invariance and covariance under special conformal transformations. Throughout the paper we keep all $x_{i}$ distinct and do not discuss those Ward Identities which involve coincident points.

${ }^{2}$ The relation between the CFT correlators and scattering amplitudes is also discussed from the holographic point of view in $[7,8]$.
} 
the four-point function of stress-energy tensors or conserved currents and propose the way to formulate the conformal bootstrap constraints without degeneracy. In section 3 we discuss Ward Identities in the momentum space and establish an interesting parallel between imposing special conformal invariance in the coordinate space and conservation in the momentum space. In section 4 we calculate the number of functions governing scattering amplitudes of massless particles in an auxiliary Minkowski space and compare it with the number of unrestricted degrees of freedom calculated in section 2 . We conclude with section 5 .

\section{Imposing conservation in the coordinate space}

In a conformal field theory the full set of Ward Identities can be understood in the following way. The underlying symmetries impose that the correlator of primary operators $\left\langle\mathcal{O}^{1}\left(x_{1}\right) \ldots \mathcal{O}^{n}\left(x_{n}\right)\right\rangle$ is a covariant function under conformal transformations of $x_{i}$. Besides, if the dimension $\Delta_{i}$ reaches the unitary bound the corresponding operator is conserved

$$
\left\langle\ldots \partial_{\mu} \mathcal{O}_{\mu \ldots}^{i} \ldots\right\rangle=0
$$

There are other W.I.'s but they are trivially satisfied when all $x_{i}$ are distinct (which we implicitly assume throughout the paper).

The covariance under conformal transformations (this also includes Poincaré symmetry) can be solved in a number of ways, in particular using the embedding formalism [6]. An explicit expression for the desired correlator will involve a number of arbitrary functions of the conformal cross-ratios. For the four-point function of the identical operators of dimension $\Delta$ it takes the form

$$
\left\langle\mathcal{O}_{\mu_{1} \ldots}^{1}\left(x_{1}\right) \ldots \mathcal{O}_{\mu_{4} \ldots}^{4}\left(x_{n}\right)\right\rangle=\prod_{i<j}^{4}\left(x_{i}-x_{j}\right)^{-2 \Delta / 3} \sum_{I}^{N} f^{I}(u, v) \mathbb{Q}_{I \mu_{1} \ldots \mu_{4} \ldots}
$$

The tensor structures $\mathbb{Q}_{I}$ are some known expressions made of $x_{i}^{\mu}$ and the flat space metric (Kronecker delta-symbol) $\delta_{\mu \nu},{ }^{3}$ while $u, v$ are defined in (1.1). More concretely, each $\mathbb{Q}$ is a product of certain "building block" tensors $H_{\mu \nu}^{(i j)}$ and $V_{\mu}^{i[j k]}$ and the total number of structures $N$ reflects the number of all possible combinations of $V$ 's and $H$ 's resulting in the desired tensor structure of $\mathbb{Q} .{ }^{4}$

Now, in a special case when $\Delta_{i}$ saturates the unitary bound (for traceless symmetric operator of spin $\ell$ it is $\Delta=\mathrm{d}+\ell-2$ ), the derivative $\partial_{\mu} \mathcal{O}_{\mu \ldots}^{i}$ has the correct property of a primary field of dimension $\Delta+1$. Therefore the correlator with $\mathcal{O}_{\mu \ldots}^{i}$ substituted by $\partial_{\mu} \mathcal{O}_{\mu \ldots}^{i}$ should have a similar representation to (2.2) albeit with a slightly altered overall prefactor and the new set of tensors $\tilde{\mathbb{Q}}_{\tilde{I}}$ and functions $\tilde{f}^{\tilde{I}}$. The new functions $\tilde{f}$ are related

\footnotetext{
${ }^{3}$ By default we are working in the d-dimensional Euclidean space $\mathbb{R}^{\mathrm{d}}$, but our results are equally valid in the Minkowski space $\mathbb{R}^{\mathrm{d}-1,1}$ after a trivial substitution $\delta_{\mu \nu} \rightarrow \eta_{\mu \nu}$. Another important comment: throughout the paper we focus on the parity-even part of the four-point functions (this does not require the theory to preserve parity). This explains absence of $\epsilon$-tensors inside $\mathbb{Q}$.

${ }^{4}$ In $(2.2)$ we slightly altered the definitions of $f^{I}(u, v)$ (by a factor of $\left(u^{2} / v\right)^{\Delta / 3}$ ) as well as $H$ and $V$ compared with [6].
} 
to the original ones through an action of some first order differential operator in variables $u, v$. Thus the conservation of $\mathcal{O}^{i}$ inside the correlator (2.2) is equivalent to $\tilde{f}^{\tilde{I}}=0$ for all $\tilde{I}$ which can be rewritten in the following way

$$
\left[A_{I}^{\tilde{I}}+B_{I}^{\tilde{I}} \frac{\partial}{\partial u}+C_{I}^{\tilde{I}} \frac{\partial}{\partial v}\right] f^{I}(u, v)=0
$$

Here $A, B, C(u, v)$ are some rectangular matrices which depend on $x_{i}^{\mu}$ only through $u$ and $v$ (these matrices also depend on the dimension $\mathrm{d}$ and a choice of the basis for $\mathbb{Q}$ 's). Conservation of each $\mathcal{O}^{i}$ inside the correlator leads to (2.3) with its own set of matrices $A, B, C$. With some effort these matrices can be calculated in each particular case (in all cases considered below we calculated $A, B, C$ explicitly using computer algebra). But unfortunately the resulting equations are complicated enough such that we could not find an explicit solution or express it in any other self-contained way. In what follows we will merely analyze these equations with the goal of calculating the number of functional degrees of freedom unconstrained by (2.3). We will carry on explaining our logic in a particular case of the correlator of four conserved currents $J_{\mu}$ and return to the four-point function of the stress-energy tensors in the end of this section.

\subsection{Conservation constraints for conserved currents}

In a case of four conserved currents ${ }^{5}$ of dimension $\Delta$ in general d there are 43 corresponding structures $\mathbb{Q}$ and 14 structures $\tilde{\mathbb{Q}}$

$$
\begin{aligned}
\left\langle J_{\mu} J_{\nu} J_{\rho} J_{\sigma}\right\rangle & =\prod_{i<j}^{4}\left(x_{i}-x_{j}\right)^{-2 \Delta / 3} \sum_{I}^{43} f^{I}(u, v) \mathbb{Q}_{I \mu \nu \rho \sigma}, \\
\left\langle\mathcal{O} J_{\nu} J_{\rho} J_{\sigma}\right\rangle & =\prod_{i<j}^{4}\left(x_{i}-x_{j}\right)^{-2 \Delta / 3-\delta_{i, 1} / 3} \sum_{\tilde{I}}^{14} \tilde{f}^{\tilde{I}}(u, v) \tilde{\mathbb{Q}}_{\tilde{I} \nu \rho \sigma} .
\end{aligned}
$$

The scalar operator $\mathcal{O}$ in $(2.5)$ is of dimension $\Delta+1$. In case $\Delta=\mathrm{d}-1$ the conservation condition for the first current $J_{\mu}$ in (2.4) will take the form (2.3) with the $14 \times 43$ matrices $A_{1}, B_{1}, C_{1}$. Similarly, the conservation condition for the second current $J_{\nu}$ would yield another 14 equations i.e. another set of matrices $A_{2}, B_{2}, C_{2}$, etc. All together, there are four conservation conditions, one for each current. Combining them all together we obtain a set of 56 equations which can be cast in the form (2.3) with some $56 \times 43$ matrices $A, B, C$. Although there are more equations then unknown functions, these equations are not independent. As a result there are unrestricted degrees of freedom which we wish to identity.

\subsection{Permutation symmetry I}

Our next step is to reduce the number of functions $f^{I}$ by imposing the permutation symmetry which changes the order of operators inside the correlator. In case the operators are

\footnotetext{
${ }^{5}$ For simplicity we assume that all four currents are identical. It is easy to generalize this by introducing a color index $J_{\mu}^{a}$, such that each $f^{I}$ will carry four such indexes $f^{I \text { abcd }}$.
} 
bosonic, any permutation should be a symmetry. Otherwise in certain cases the correlator may change sign. For the four point function there are $4 !=24$ possible permutations (including the trivial one). But the following $\mathbb{Z}_{2} \times \mathbb{Z}_{2}$ subgroup of $\mathbf{S}_{4}$ that consists of the following permutations

$$
(1234) \rightarrow(2143), \quad(1234) \rightarrow(3412), \quad(1234) \rightarrow(4321)
$$

is of particular importance: these permutations leave the cross-ratios $u, v$ invariant. The action of these permutations on the functions $f^{I}(u, v)$ is purely algebraic: $f^{I}(u, v) \rightarrow$ $f_{\text {new }}^{I}(u, v)=S_{J}^{I}(u, v) f^{J}(u, v)$. Invariance of $\left\langle J_{\mu} J_{\nu} J_{\rho} J_{\sigma}\right\rangle$ thus reduced to a linear algebra problem of finding the kernel of $\left(\delta_{J}^{I}-S_{J}^{I}(u, v)\right)$. We will use the same notations $f^{I}(u, v)$ to denote vectors from this kernel in some unspecified basis, although now $I$ would run up to 19 - the dimension of the kernel in the particular case of four conserved currents. ${ }^{6}$

Invariance with respect to $\mathbb{Z}_{2} \times \mathbb{Z}_{2}$ allows us to reduce the number of the unknown functions from 43 to 19 . Moreover the number of linearly independent equations reduces to 14 . Indeed, the permutations (2.6) are just enough to bring any current out of four to the first position. Hence it is enough to impose conservation of the first current only, all other conservation conditions will be linearly dependent. To conclude, imposing invariance under (2.6) restricts the problem to the set of equations of the form (2.3) with some $14 \times 19$ matrices $A, B, C$ and 19 functions $f^{I}(u, v)$. In what follows we will refer to these equations as the "conservation constraints".

Let us briefly explain the effect of other permutations. They act on $f^{I}$ by a combination of linear transformation and a change of variables. For example the permutation

$$
(1234) \rightarrow(2134)
$$

maps $(u, v)$ into $\left(u^{\prime}, v^{\prime}\right) \equiv(u / v, 1 / v)$ and $f^{I}(u, v)$ into

$$
f_{\text {new }}^{I}(u, v)=S_{J}^{I}(u, v) f^{J}\left(u^{\prime}, v^{\prime}\right)
$$

The square matrix $S$ is such that if $f^{I}(u, v)$ satisfy the conservation constraints, $f_{\text {new }}^{I}(u, v)$ will also do so.

\subsection{Number of unrestricted functional degrees of freedom}

Now it is time to return to the conservation constraints (2.3) and calculate the number of functional degrees of freedom unconstrained by these equations. This can be done using the following simple trick. ${ }^{7}$ Let us rename the variables $u, v$ into $t, x$ and think of $t$ as "time" and $x$ as "space" coordinate. Next, we would like to think of (2.3) as a Cauchy problem, namely consider (2.3) as a set of algebraic equations on the "time" derivatives $\partial f^{I} / \partial t$ which we need to express in terms of the original functions $f^{I}$ and spatial derivatives $\partial f^{I} / \partial x$.

\footnotetext{
${ }^{6}$ Strictly speaking the kernel is parametrized by some new $g^{K}(u, v)$ with $K=1 \ldots 19$, while the nineteen permutation-invariant vectors $f^{I}(u, v), I=1 \ldots 43$, are linear combinations of $g^{K}$. In order to avoid the notation clutter in what follows we rename $g^{K}$ into $f^{I}$ and hope this will not cause any confusion.

${ }^{7}$ We thank Vasily Pestun for suggesting this idea.
} 
The $14 \times 19$ matrix $B(t, x)$ has rank 12 which means the conservation constraints $(2.3)$ can be rewritten as twelve "time evolution" equations

$$
\frac{\partial f^{i}}{\partial t}=F^{i}\left[f^{J}, \frac{\partial f^{J}}{\partial x}\right]
$$

for certain twelve $f^{i}$ (say, $i=1 \ldots 12$ ) and two constraints "without time derivatives"

$$
G_{1,2}\left[f^{J}, \frac{\partial f^{J}}{\partial x}\right]=0
$$

It can be checked that the constraints (2.10) are of the first type. Now one can think of (2.9), (2.10) as of Cauchy boundary problem for $f^{i}$ while the remaining $f^{\alpha}$ with $\alpha=$ $13 \ldots 19$ are unrestricted and should be thought of as the external parameters. The "initial conditions" are specified by the boundary values $f^{i}\left(t^{*}, x\right)$ at some boundary $t=t^{*}$ such that the constraints (2.10) are satisfied.

Our first conclusion is that the bose symmetric four-point function of the conserved currents in a general CFT is governed by seven functional degrees of freedom (we called them $f^{\alpha}$ above). It is important to note that this number is well-defined and will not change upon a new choice of "time" direction in the $(u, v)$-plane: the rank of any linear combination of $B$ and $C$ is always $12 .{ }^{8}$

Besides seven unrestricted functions, the correlator also depends on the "initial conditions" $f_{i}\left(t^{*}, x\right)$ at some $t=t^{*}$. Furthermore we still have to impose bose symmetry with respect to the permutations that change $(u, v)$. There are $3 !=6$ of those corresponding to the $\mathbf{S}_{3}$ group that keeps the first operator inside the correlator in its place and permutes the other three. We will impose this symmetry in the next subsection.

\subsection{Permutation symmetry II}

Although the equations (2.3) are defined for any $u$ and $v$, in a Euclidean theory $u$ and $v$ are non-negative, see (1.1). In fact the physically accessible points must lie inside the curve $\sqrt{u}+\sqrt{v}=1$ (this area is highlighted in color in figure 1a). This fact does not invalidate our previous findings based on the Cauchy problem picture on the whole $u, v$ plane (or a quadrangle $u, v \geq 0$ ) because we can think of the conservation constraints mathematically, without worrying about physical origin and hence scope of $u$ and $v$ (it is important to note that nothing special happens to $A, B, C$ on the boundary $\sqrt{u}+\sqrt{v}=1)$.

The "physical" area consists of six patches (each highlighted in its own color in figure 1a) which are mapped into each other by $\mathbf{S}_{3}$. The permutation symmetry constraints $f^{I}(u, v)=f_{\text {new }}^{I}(u, v)$ equate $f^{I}$ from different patches. Thus, at least conceptually, it is enough to know $f^{I}$ just in one patch and require that $f^{I}$ satisfy certain conditions on the patch's boundary (which sometimes is mapped into itself under $\mathbf{S}_{3}$ ) to ensure permutation symmetry.

Unless the "time" and "space" coordinates are chosen wisely the permutation symmetry would mix the unrestricted $f^{\alpha}$ and dependent $f^{i}$ degrees of freedom. Therefore

\footnotetext{
${ }^{8}$ This follows from the explicit form of matrices $B, C$.
} 


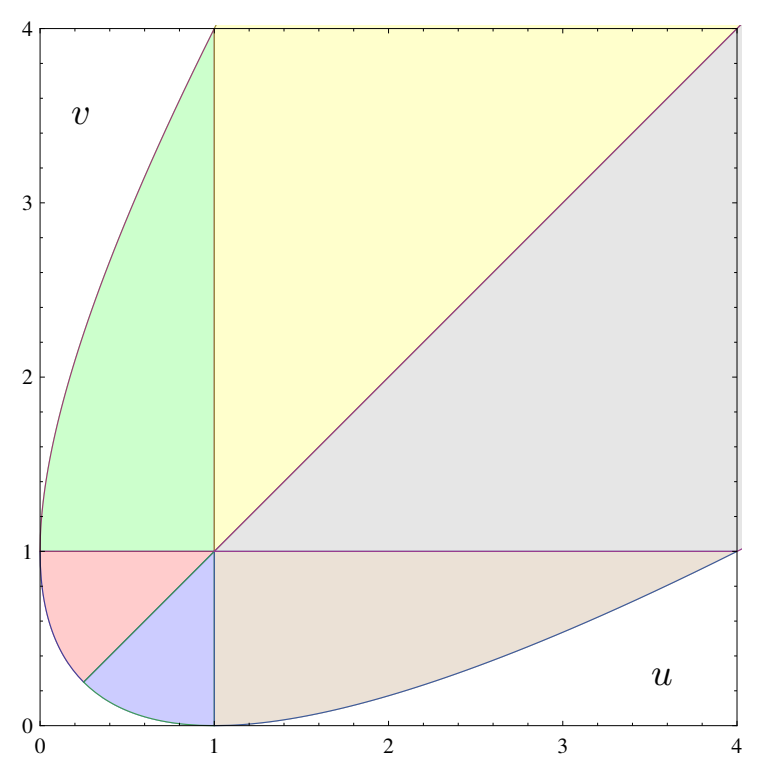

a).

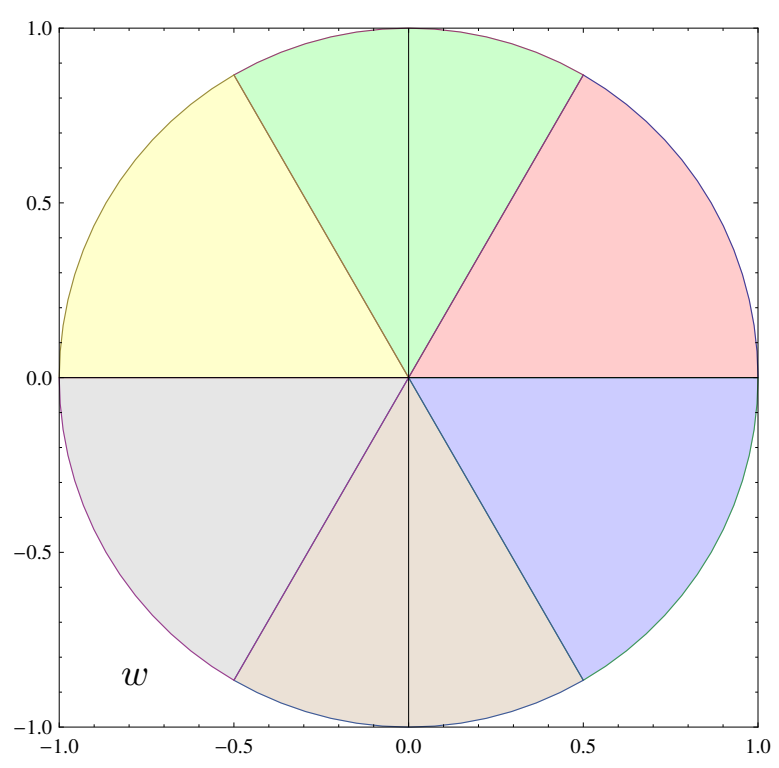

b).

Figure 1: (a) The $(u, v)$-plane with physically accessible area of cross-ratios highlighted in color. (b) The same area after a change of variables $(u, v) \rightarrow \omega$.

the permutation symmetry constraints $f^{I}(u, v)=f_{\text {new }}^{I}(u, v)$ will necessarily involve all of them. This is definitely not the most concise and desirable way. This complication can be avoided if we choose "time" coordinate coordinate $t$ such that all permutations will map it into itself $\mathbf{S}_{3}:(t, x) \rightarrow\left(t, x^{\prime}\right)$ where $x^{\prime}$ is some function of $(t, x)$. In such a case the boundary $t=t^{*}$ will be mapped into itself, and intuitively we expect the sets of $f^{i}$,s and $f^{\alpha}$ 's to remain invariant (although $f^{i}$ 's and $f^{\alpha}$ 's would mix between themselves). Indeed, let us rewrite the conservation constraints in the following form

$$
\left[\mathcal{A}_{J}^{\tilde{I}}+\mathcal{B}_{J}^{\tilde{I}} \frac{\partial}{\partial t}+\mathcal{C}_{J}^{\tilde{I}} \frac{\partial}{\partial x}\right] f^{J}=0
$$

Here we use $\mathcal{A}, \mathcal{B}, \mathcal{C}$ instead of $A, B, C$ to emphasize that we changed variables $u, v$ into $t, x$ and hence the former are some linear combinations of the latter. For any values of $t, x$ the matrix $\mathcal{B}(t, x)$ has a seven-dimensional kernel which we parametrize by introducing basis elements $\xi_{\alpha}^{I}(t, x): \mathcal{B}_{J}^{I} \xi_{\alpha}^{J}=0$. We denote other twelve linearly independent vectors spanning the space of $f^{I}(t, x)$ by $\zeta_{i}^{I}(t, x)$ :

$$
f^{I}=f^{i} \zeta_{i}^{I}+f^{\alpha} \xi_{\alpha}^{I} .
$$

The linear space spanned by $\zeta$ 's is not well-defined because one could shift $\zeta$ 's by $\xi$ 's. A good way to remove this ambiguity is to introduce a positive-definite metric $g_{I J}(t, x)$ and require orthogonality of all $\zeta$ 's and $\xi$ 's (i.e. $\zeta$ 's will span the orthogonal complement to the kernel of $\mathcal{B})$.

Crucially, we will assume that $g_{I J}(t, x)$ is covariant under the permutation symmetry (now we switch back from $(t, x)$ to $(u, v)$ to stress that covariance of metric is independent 
of the choice of variables)

$$
S_{I^{\prime}}^{I}(u, v) g_{I J}(u, v) S_{J^{\prime}}^{J}(u, v)=g_{I^{\prime} J^{\prime}}\left(u^{\prime}, v^{\prime}\right) .
$$

Going back to $(2.12)$ we define $\zeta$ 's such that $\zeta_{i}^{I}(u, v) g_{I J}(u, v) \xi_{\alpha}^{J}(u, v)=0$ for any $i, \alpha$.

In section 2.2 we explained that $f_{\text {new }}^{I}$ automatically solves $(2.11)$ so far $f^{I}$ does. This can only happen if the equations resulting from plugging (2.8) into (2.11) are linearly dependent with the original equations (2.11) upon a change of variables $u, v \rightarrow u^{\prime}, v^{\prime}$. In particular this means (this crucially uses that "time" $t$ is mapped into itself: $t^{\prime}=t$ )

$$
\mathcal{B}^{\tilde{I}}(u, v)_{J} S_{J^{\prime}}^{J}(u, v)=\mathbf{s}_{\tilde{I}^{\prime}}^{\tilde{I}}\left(u^{\prime}, v^{\prime}\right) \mathcal{B}_{J^{\prime}}^{\tilde{I}^{\prime}}\left(u^{\prime}, v^{\prime}\right),
$$

for some matrix s. From here it immediately follows that $S_{J}^{I}(u, v) \xi_{\alpha}^{J}\left(u^{\prime}, v^{\prime}\right)$ is annihilated by $\mathcal{B}_{J}^{\tilde{I}}(u, v)$ and hence it can be expanded in a linear combination of $\xi$ 's:

$$
S_{J}^{I}(u, v) \xi_{\alpha}^{J}\left(u^{\prime}, v^{\prime}\right)=\xi_{\beta}^{I}(u, v) \lambda_{\alpha}^{\beta}(u, v),
$$

for some matrix $\lambda$. In short, we just derived that the space of $\xi$ 's is invariant under permutations from $\mathbf{S}_{\mathbf{3}}$. Since $\zeta$ 's were defined as a basis in the orthogonal complement to $\xi$ 's and the metric is covariant under $\mathbf{S}_{\mathbf{3}}$ we conclude that there is a matrix $w$ such that $S_{J}^{I}(u, v) \zeta_{i}^{J}\left(u^{\prime}, v^{\prime}\right)=\zeta_{j}^{I}(u, v) w_{i}^{j}(u, v)$.

Now the permutation symmetry constraint $f^{I}(u, v)=f_{\text {new }}^{I}(u, v)$ can be rewritten as two separate conditions: one for the unrestricted degrees of freedom $f^{\alpha}$

$$
\lambda_{\beta}^{\alpha}(u, v) f^{\beta}\left(u^{\prime}, v^{\prime}\right)=f^{\alpha}(u, v),
$$

and a similar one for $f^{i}$ s

$$
w_{j}^{i}(u, v) f^{j}\left(u^{\prime}, v^{\prime}\right)=f^{i}(u, v)
$$

\subsection{Conformal bootstrap for unrestricted D.O.F.}

Conformal bootstrap for a four-point functions is a combination of two basic properties of a CFT correlator: crossing symmetry and conformal block decomposition. The latter is just the statement that the corresponding functions $f^{I}(u, v)$ is a linear combination of some special predetermined functions $G_{\Delta, \ell, \ldots}^{I}(u, v)$ universal for all CFTs in a given dimension $\mathrm{d}$, which are called conformal blocks ${ }^{9}$

$$
f^{I}(u, v)=\sum_{\Delta, \ell, \ldots} c_{\Delta, \ell, \ldots} G_{\Delta, \ell, \ldots}^{I}(u, v)
$$

Here coefficients $c_{\Delta, \ell, \ldots}$ are the products of the CFT structure constants that fix the value of the three-point functions. Quantum numbers $\Delta$ and $\ell$ denote dimension and Lorentz representation (called spin for brivety) of the intermediary operators $\mathcal{O}_{\Delta, \ell}$. The dots stand for other quantum numbers counting different possible forms of the three-point functions of the operator $\mathcal{O}_{\Delta, \ell}$ with the operators $\mathcal{O}_{\mu_{i} \ldots}^{i}$ of (2.2). In case $\mathcal{O}_{\mu_{i} \ldots}^{i}$ are conserved the

\footnotetext{
${ }^{9}$ Our definition of conformal blocks could differ from the conventional one by a fixed prefactor.
} 
corresponding three-point functions are assumed to be conserved as well, restricting the number of different $c_{\Delta, \ell, \ldots}$.

The crossing symmetry is the symmetry of the corresponding correlator under permutations of the operators. In other words crossing symmetry is summarized in two conditions (2.16) and (2.17) derived above. Since $f^{i}$ 's depend on $f^{\alpha}$ 's we suspect the second set of conditions (2.17) is redundant. Strictly speaking we can not prove that because besides $f^{\alpha}$ 's functions $f^{i}(t, x)$ also depend on the boundary conditions $f^{i}\left(t^{*}, x\right)$. At the same time we believe in a physical theory where $f^{I}$ satisfy the conformal block decomposition (2.18) functions $f^{\alpha}(u, v)$ completely and unambiguously determine $f^{i}$ and hence (2.17) follows from (2.16). Thus the conformal bootstrap can be conveniently formulated in terms of only unrestricted degrees of freedom as follows

$$
\begin{aligned}
& f^{\alpha}(u, v)=\sum_{\Delta, \ell, \ldots} c_{\Delta, \ell, \ldots} G_{\Delta, \ell, \ldots}^{\alpha}(u, v), \\
& f^{\alpha}(u, v)=\lambda_{\beta}^{\alpha}(u, v) f^{\beta}\left(u^{\prime}, v^{\prime}\right) .
\end{aligned}
$$

Here we introduced $\xi_{I}^{\alpha}(u, v)$ such that $\xi_{I}^{\alpha} \xi_{\beta}^{I}=\delta_{\beta}^{\alpha}, \xi_{I}^{\alpha} \zeta_{i}^{I}=0$ and

$$
f^{\alpha}=\xi_{I}^{\alpha} f^{I}, \quad G^{\alpha}=\xi_{I}^{\alpha} G^{I}
$$

The covectors $\xi_{I}^{\alpha}$ satisfying necessary properties can be constructed from $\xi_{\alpha}^{I}$ and $g_{I J}$,

$$
\xi_{I}^{\alpha}=g^{\alpha \beta} g_{I J} \xi_{\beta}^{J}, \quad g_{\alpha \beta}=\xi_{\alpha}^{I} g_{I J} \xi_{\beta}^{J} .
$$

More generally one can consistently formulate the bootstrap equations (2.19), (2.20), (2.21) with help of any set of covectors $\xi_{I}^{\alpha}(u, v)$ which is closed under permutations, i.e. such that there is an appropriate $\lambda_{\beta}^{\alpha}(u, v)$ satisfying

$$
\xi_{I}^{\alpha}(u, v) S_{J}^{I}(u, v)=\lambda_{\beta}^{\alpha}(u, v) \xi_{J}^{\beta}\left(u^{\prime}, v^{\prime}\right) .
$$

We expect that any additional bootstrap equations would be redundant as soon as the number of linearly-independent $\xi_{I}^{\alpha}$ reaches the number of unrestricted functional degrees of freedom governing the corresponding correlation function (see table 1).

The permutation-covariant set $\xi_{I}^{\alpha}$ can be conveniently constructed through (2.22) starting from any set of vectors $\xi_{\alpha}^{I}$ which by itslef is closed under permutations, i.e. which satisfies (2.15) for some appropriate $\lambda_{\alpha}^{\beta}(u, v)$. In section 2.4 we defined $\xi_{\alpha}^{I}$ as a basis of kernel of $\mathcal{B}_{J^{\prime}}^{\tilde{I}^{\prime}}$ corresponding to some permutation-invariant coordinate $t$. Alternatively one could use a particular form of the CFT correlator in some exactly-solvable (free) theory or the form of the correlator in special kinematics to construct the permutation covariant set of $\xi_{\alpha}^{I}$ 's. Finally, one can start with arbitrary vector $\xi^{I}$ and act by permutations until obtaining a closed set $\xi_{\alpha}^{I}$.

Certainly our formulation of the bootstrap equations in term of only unrestricted degrees of freedom (2.19), (2.20), (2.21) would remain just a hypothetical idea unless we can provide the necessary building blocks: permutation covariant positive-definite metric 
$g_{I J}$ and a coordinate system $t, x$ such that $t$ is permutation invariant. In fact such a metric can be easily constructed by contracting all Lorentz indexes of $Q$ 's:

$$
g_{I J}(u, v)=Q_{I \mu \nu \ldots}\left(x_{i}\right) Q_{J \mu \nu \ldots}\left(x_{i}\right) .
$$

Similarly, finding such $t$ is not very difficult. For example for any function $g(u)$ a sum over all permutations $\sum_{\mathbf{a} \in \mathbf{S}_{\mathbf{3}}} g(\mathbf{a}(u))$ or any function of this expression will be permutation invariant, for example

$$
t=u+v+\frac{u}{v}+\frac{v}{u}+\frac{1}{u}+\frac{1}{v} .
$$

A general $t$ complimented by some $x$ would lead to a very complicated $\xi$ and $\lambda$ rendering our scheme (2.19), (2.20), (2.21) impractical. To deal with this problem we propose the following coordinate system which incorporates symmetries of the problem in a most natural elegant way. ${ }^{10}$ We rename $t, x$ into $r, \phi$ and introduce $\omega=r e^{i \phi}$ through

$$
u=\left|\frac{1+\gamma \omega}{1+\omega}\right|^{2}, \quad v=\left|\frac{1+\bar{\gamma} \omega}{1+\omega}\right|^{2}, \quad \gamma=e^{-2 \pi i / 3} .
$$

The new variable $\omega$ is related to the canonical variable $z$ defined through $u=z \bar{z}, v=$ $(1-z)(1-\bar{z})$ through a Möbius transform

$$
z=(1+\bar{\gamma}) \frac{1+\gamma \omega}{1+\omega}
$$

The transformation $(u, v) \rightarrow \omega$ maps the "physical" area in the $u, v$-plane, figure 1a, into a "pizza pie" - the unit disk on the complex plane split into six equal patches ("slices"), figure $1 \mathrm{~b}$. The permutation symmetry $\mathbf{S}_{\mathbf{3}}$ acts on $\omega$ "canonically" according to a naive geometrical intuition i.e. "slices" are permuted while radius $r$ is left intact. The two generators of $\mathbf{S}_{\mathbf{3}}$ could be chosen to be $\phi \rightarrow-\phi$ and $\phi \rightarrow 2 \pi / 3-\phi$.

\subsection{Small d and degenerate tensors}

Above we have calculated the number of unrestricted functional degrees of freedom governing the four-point function of conserved currents $\left\langle J_{\mu} J_{\nu} J_{\rho} J_{\sigma}\right\rangle$. The calculation (and the result — seven kinematically-unrestricted functional D.O.F.) was seemingly independent on the dimension d. This is not entirely correct: the tensors $\mathbb{Q}_{I}$ and $\tilde{\mathbb{Q}}_{\tilde{I}}$ from (2.4) and (2.5) as well as matrices $A, B, C$ depend on d. Thus it might happen that some of the properties used in our calculation, such as rank of $\mathcal{B}$, are different in some specific dimensions from the generic values. This does not happen in all cases we studied, but there is another subtlety associated with small d: tensors $\mathbb{Q}_{I}$ (and/or $\tilde{\mathbb{Q}}_{\tilde{I}}$ ) could become degenerate. We discuss when this may occur in appendix A. Here we just note that degeneracy of $\mathbb{Q}_{I}$ would mean that certain linear combination $(\mathrm{s}) \xi_{0}^{I}(u, v) \mathbb{Q}_{I}$ vanish identically for any values of $x_{i}^{\mu}$. Correspondingly the metric $g_{I J}$ is degenerate in this case, in particular $g_{I J} \xi_{0}^{J}=0$. This is exactly what happens in $d=3$ : two combinations of $\mathbb{Q}_{I}$ 's are identically zero. Hence two out of seven unrestricted functions are in fact unphysical: they could be chosen to be

\footnotetext{
${ }^{10}$ We thank Sungjay Lee for help with finding $\omega$.
} 
anything (for example zero) without affecting $\left\langle J_{\mu} J_{\nu} J_{\rho} J_{\sigma}\right\rangle$. Hence in $\mathrm{d}=3$ the number of unrestricted functional degrees of freedom is in fact five, not seven.

Let us briefly discuss how the presence of degenerate $\mathbb{Q}_{I}$ 's would affect the bootstrap equations. Since $\xi_{0}^{I} \mathbb{Q}_{I}=0$ identically for all $x_{i}^{\mu}$ we should choose $\xi_{I}^{\alpha}$ in (2.21) such that it is orthogonal to all degenerate $\xi_{0}^{I}, \xi_{I}^{\alpha} \xi_{0}^{I}=0$. This would automatically be so if we start with a set of vectors $\xi_{\alpha}^{I}$ closed under permutations and define

$$
\xi_{I}^{\alpha}=g_{I J} \xi_{\alpha}^{J}
$$

The only difference with (2.22) here is that we did not introduce the metric $g_{\alpha \beta}$ because it might be degenerate. If $\xi_{\alpha}^{I}$ were closed under permutations so would be $\xi_{I}^{\alpha}$, although not all of them might be linearly independent. This is what happens for four currents in $d=3$ : starting from seven $\xi_{\alpha}^{I}$ one finds only five linearly-independent $\xi_{I}^{\alpha}$. To formulate the bootstrap equations one should take the set of all linearly-independent $\xi_{I}^{\alpha}$ and find $\lambda_{\alpha}^{\beta}$ satisfying (2.23). Then the bootstrap equations are readily given by (2.19), (2.20), (2.21).

It was important for us above that while some of $\mathbb{Q}_{I}$ 's were degenerate, all $\tilde{\mathbb{Q}}_{\tilde{I}}$ were linearly independent. Otherwise instead of equating (2.3) to zero we would have to allow arbitrary functions of $u, v$ along certain directions in $\tilde{I}$ in the r.h.s. . This would complicate counting the unrestricted degrees of freedom and we avoid discussing such cases. To summarize, parity-even part of the correlation function of four conserved currents in a general CFT is governed by five in $d=3$ and seven in $d \geq 4$ functional degrees of freedom. ${ }^{11}$

\subsection{Four point function of the stress-energy tensors}

Eventually we are ready to return to the four-point function of the stress-energy tensors. This case is very similar to the four conserved currents discussed above, but the involved matrices are much larger. Thus generically the four-point function of $T_{\mu \nu}$ 's involves 633 independent $\mathbb{Q}_{I}$ 's:

$$
\begin{aligned}
\left\langle T_{\mu_{1} \nu_{1}} T_{\mu_{2} \nu_{2}} T_{\mu_{3} \nu_{3}} T_{\mu_{4} \nu_{4}}\right\rangle & =\prod_{i<j}^{4}\left(x_{i}-x_{j}\right)^{-2 \Delta / 3} \sum_{I}^{633} f^{I}(u, v) \mathbb{Q}_{I \mu_{1} \ldots \nu_{4}}, \\
\left\langle J_{\nu_{1}} T_{\mu_{2} \nu_{2}} T_{\mu_{3} \nu_{3}} T_{\mu_{4} \nu_{4}}\right\rangle & =\prod_{i<j}^{4}\left(x_{i}-x_{j}\right)^{-2 \Delta / 3-\delta_{i, 1} / 3} \sum_{\tilde{I}}^{302} \tilde{f}^{\tilde{I}}(u, v) \tilde{\mathbb{Q}}_{\tilde{I} \nu_{1} \ldots \nu_{4}} .
\end{aligned}
$$

Here $T_{\mu \nu}$ is an abstract spin two traceless symmetric primary of dimension $\Delta$ and $J_{\nu}$ is a primary vector of dimension $\Delta+1$. In reality $\Delta=\mathrm{d}$ and $T_{\mu \nu}$ is conserved which results in the equation $(2.3)$ with $(4 \times 302) \times 633$ matrices $A, B, C$.

Imposing symmetry under permutations (2.6) results in the space of $f^{I}$ being reduced from 633 to 201 functions: now the matrices $(2.3)$ are $302 \times 201$. Given that matrix $B$ (or any linear combination of $B$ and $C$ ) has rank 172 the number of unrestricted functions is 29. This a general result valid for $\mathrm{d} \geq 6$ when all $\mathbb{Q}_{I}$ 's and $\tilde{\mathbb{Q}}_{\tilde{I}}^{\prime}$ 's are linearly independent. In $\mathrm{d}=5$ while all $\tilde{\mathbb{Q}}_{\tilde{I}}$ 's are distinct one combination of $\mathbb{Q}_{I}$ 's vanishes, hence the number

\footnotetext{
${ }^{11}$ Throughout this paper we assume $\mathrm{d} \geq 3$. The case of $\mathrm{d}=2$ would involve too many degeneracies and is much easier to analyze using different formalism [22, 23].
} 
of unrestricted functional D.O.F. reduced to 28 . For $\mathrm{d}=3,4$ there are many degenerate $\tilde{\mathbb{Q}}_{\tilde{I}}$ 's (see appendix A) which complicates further analysis. We will calculate the number of unrestricted functions in this case in the next section using "duality" between the CFT correlators and scattering amplitudes in an auxiliary Minkowski space.

\section{CFT correlators and scattering amplitudes}

It was first noticed in [5] in a particular case of stress-energy tensors and further generalized in [6] that the number of linearly independent three-point functions of any (conserved or not) primary operators $\mathcal{O}_{i}$ of spin $\ell_{i}$ (for simplicity we are talking only about traceless symmetric representations; spin $\ell_{i}$ is just the number of indexes) in a general d-dimensional CFT coincides with the number of linearly independent scattering amplitudes of "dual" particles of spin $\ell_{i}$ in a $d+1$-dimensional Minkowski space. When some operators are conserved, i.e. when the corresponding $\Delta_{i}$ saturate the unitary bound, the dual particles should be massless $p_{i}^{2}=0$. Otherwise $p_{i}^{2} \neq 0$.

In [6] this intriguing coincidence was given the following interpretation. The threepoint scattering amplitudes ${ }^{12}$ in a flat space $\mathbb{R}^{\mathrm{d}, 1}$ can be one-to-one matched with the cubic interacting vertexes in the Lagrangian. Next, these vertexes are brought into the $A d S_{\mathrm{d}+1}$ space, where through the usual AdS/CFT logic, they give rise to the CFT correlators at the boundary $\mathbb{R}^{\mathrm{d}-1,1}$ (or $\mathbb{R}^{\mathrm{d}}$ upon a Wick rotation). This picture works well for the threepoint functions of any (conserved or not) primaries but its validity and completeness for the four (and higher)-point functions is not clear. Thus the $2 \rightarrow 2$ scattering amplitude is not completely determined by the quartic coupling in the Lagrangian, rather it depends on all cubic couplings in the theory. So is the four-point function on the boundary of AdS - it also depends on all cubic couplings in the bulk. Hence one can envision a matching procedure between the four-point scattering amplitudes in $\mathbb{R}^{\mathrm{d}, 1}$ and the four-point CFT correlators in $\mathbb{R}^{\mathrm{d}}$, but at this point this has not been done.

Nevertheless it was proved in [6] that the number of functional degrees of freedom governing $n$-point functions of primary operators $\mathcal{O}_{i}$ with all $\Delta_{i}$ above the unitary bound (i.e. no $\mathcal{O}_{i}$ is conserved) is indeed equal to the number of functions governing scattering amplitudes of $n$ "dual" massive particles in $\mathbb{R}^{\mathrm{d}, 1}$. Let us remind the reader how this was established. When all $\mathcal{O}_{i}$ are non-conserved a general $n$-point function is given by some generalization of (2.2) with all functions $f^{I}$ (which depend on $n(n-3) / 2$ conformal cross-ratios) being unconstrained. The corresponding tensors $\mathbb{Q}_{I}$ 's satisfy certain linear conditions and can be constructed as all possible products of "building blocks" $H_{\mu \nu}^{(i j)}$ and $V_{\mu}^{i[j k]}$ such that the resulting tensors have the desired set of space-time indexes and satisfy necessary symmetries.

Similarly the generic scattering amplitude of $n$ massive particles can be expressed in terms of a sum (2.2)

$$
\mathcal{A}=\sum_{\mathrm{l}} \mathrm{f}^{\mathrm{l}} \mathbb{A}_{\mathrm{I} M \ldots}
$$

\footnotetext{
${ }^{12}$ In case the involved particles are massless $1 \rightarrow 2$ process is prohibited due to kinematics and scattering amplitude in the Minkowski space $\mathbb{R}^{\mathrm{d}, 1}$ is ill-defined. In such a case the analytic continuaton in momenta into $\mathbb{R}^{\mathrm{d}-1,2}$ space is assumed. We thank Jared Kaplan and Leonardo Rastelli for discussing this point.
} 
Here $\mathrm{f}^{\prime}$ are the functions of $n(n-3) / 2$ Mandelstam variables. The tensors $A_{\mathrm{l}}$ are build of particle momenta $p_{i}^{M}$ and Kronecker delta-symbols $\delta_{M N},{ }^{13}$ and satisfy the following equivalence condition (index $M$ corresponds to the $i$-th operator $\mathcal{O}_{i}$ )

$$
\mathbb{A}_{\ldots M \ldots} \simeq \mathbb{A}_{\ldots M \ldots}+p_{M}^{i}(\ldots) .
$$

It turns out that such $\mathbb{A}_{\text {I }}$ can be also constructed as all possible products of some $H_{M N}^{(i j)}$ and $V_{M}^{i[j k]}$ (which have the same symmetries as $H_{\mu \nu}^{(i j)}$ and $V_{\mu}^{i[j k]}$ ). Since $\mathbb{A}_{l}$ 's should have the same index structure as $\mathbb{Q}$ 's we conclude that the spaces of $\mathbb{Q}_{I}$ 's and $\mathbb{A}_{I}$ 's are isomorphic. Although we started with two different sets of linear algebra constraints acting in two different linear spaces, they define isomorphic linear spaces spanned by $\mathbb{Q}_{I}$ 's or $\mathbb{A}_{I}$ 's. As a result there are as many $f^{I}$ 's as $\mathrm{fl}^{\mathrm{l}}$ 's (and that's why we can use $I$ instead of I).

It is only natural now to ask if this relation holds in case when some $\mathcal{O}_{i}$ are conserved. ${ }^{14}$ Let us stress that in this case we are no longer comparing two linear algebra problems. While the number of independent scattering amplitudes (i.e. functions $f^{\prime}$ ) is still governed by linear algebra, the unrestricted $f^{I}$ 's are controlled by the differential constraint (2.3). We could hardly do the comparison in full generality for $n$-point functions, but it is straightforward to cover the correlators of four conserved currents or stress-energy tensors. Indeed, the number of independent $f^{I}$ 's was calculated in the previous section (we covered $\mathrm{d} \geq 3$ for conserved currents and $\geq 5$ for stress-energy tensors). The number of linearly independent $\mathbb{A}_{\text {I }}$ can be calculated directly using their definition and properties: the equivalence condition (3.2) and transversality $p_{i}^{M} \mathbb{A}_{\ldots . . .}=0$. Besides, we also impose symmetry with respect to all permutations (this condition should be relaxed in case one is interested non-bose-symmetric CFT correlators). Remarkably, but not totally unexpectedly, the results of two calculations perfectly match. Thus we extend and confirm the conjecture of $[5,6]$ to include four-point functions of conserved operators. This gives us a reason to believe the relation between the CFT correlators and scattering amplitudes holds beyond the four-point function, for any combination of primaries, conserved or not.

Strictly speaking, we have only established that the number of scattering amplitudes matches the number of CFT correlators. We did not provide any meaningful map between the two spaces. But we have little doubt the observed duality is not accidental. Rather it is based on some not yet fully understood physical picture. And therefore such a map must exist, although we expect it to be nontrivial. This is because it will equate a solution to some linear algebra problem with a solution to a set of some non-trivial differential constraints. This prompts us to conjecture that there must be a better formalism to write down the CFT correlators which would not only automatically solve the conformal W.I.'s (like the embedding formalism) but also take care of the conservation constraints (whenever conserved operators are present) reducing them to linear algebra. Presumably this hypothetical formalism would be the right language to study other properties of CFT correlators, e.g. impose bootstrap constraints etc.

\footnotetext{
${ }^{13}$ Let us remind the reader that we focus on the parity-even part of the correlation functions or scattering amplitudes. Relaxing this constraint, i.e. allowing $\mathbb{Q}$ 's and $\mathbb{A}$ 's to include $\epsilon$-tensors would not change the conclusion.

${ }^{14}$ We thank Simone Giombi for posing this question.
} 


\begin{tabular}{|c|c|c|c|c|}
\hline correlator & $\mathrm{d}=3$ & $\mathrm{~d}=4$ & $\mathrm{~d}=5$ & $\mathrm{~d} \geq 6$ \\
\hline$\langle J J J J\rangle$ & 5 & 7 & 7 & 7 \\
\hline$\langle T T T T\rangle$ & 5 & 22 & 28 & 29 \\
\hline
\end{tabular}

Table 1: Number of functional degrees of freedom governing bose-symmetric four-point function of conserved currents or stress-energy tensors in a d-dimensional CFT.

We summarize our findings in table 1 (for the stress-energy tensors in $d=3,4$ the results are obtained with help of scattering amplitudes only; all other entires are calculated using both approaches: the CFT correlators and the scattering amplitudes). It is quite exciting that a very large original number of $f^{I}$ 's is distilled into a relatively small number of unrestricted functions. Thus in $\mathrm{d}=3$ there are just five of those. This strongly suggests that formulating bootstrap constraints in terms of only unrestricted degrees of freedom is not only a feasible task but also a more practical approach to bootstrap then working with all $f^{I}$ 's.

In conclusion let us briefly discuss yet another method to calculate the number of unrestricted functional degrees of freedom governing the four-point function in an abstract CFT. ${ }^{15}$ The idea is to use the conformal block decomposition (2.18) of the correlator (2.2). The sum in (2.18) goes over quantum numbers of the "intermediate" primary $\mathcal{O}_{\Delta, \ell, \mathrm{k}}$, i.e. dimension and Lorentz group representation which we schematically denoted by the total number of space-time indexes $\ell$ and other quantum number(s) $k$ (in (2.18) instead of $k$ we simply put dots). The values of $\Delta, \ell$ are unbounded from above and schematically the sum over two discrete variables $\Delta, \ell$ is responsible for the fact that $f^{I}$ depend on two variables $u, v$. For each $\mathrm{k}$ and general $\Delta, \ell$ we denote by $n_{\mathrm{k}}^{1}$ the number of linearly independent threepoint functions the operator $\mathcal{O}_{\Delta, \ell, \mathrm{k}}$ can form with $\mathcal{O}^{1}, \mathcal{O}^{2}$ and similarly $n_{\mathrm{k}}^{2}$ for $\mathcal{O}^{3}, \mathcal{O}^{4}$. Then the number of independent functional D.O.F. governing the four-point correlator will be given by the sum $\sum_{\mathrm{k}} n_{\mathrm{k}}^{1} n_{\mathrm{k}}^{2}$. We illustrate how this formula works in case of four conserved currents in $\mathrm{d}=3$ in the appendix $\mathrm{B}$.

\section{Two easy ways to solve the conservation constraints}

We have seen in section 2 that imposing conservation in the coordinate space after taking care of the conformal symmetry leads to a complicated differential constraint. At the same time the intriguing connection with the linear algebra problem of scattering amplitudes discussed in section 3 suggests there should be a better way of solving the whole set of Ward Identities, including conservation of operators. In this section we discuss two straightforward ideas to explicitly solve the conservation constraints.

\subsection{Solving all W.I.'s automatically}

In section 3 we expressed a hope that there should be a mathematically elegant way to solve all Ward Identities including conservation. Indeed, below we present a way to accomplish

\footnotetext{
${ }^{15}$ We thank Juan Maldacena and João Penedones for explaining this point to us.
} 
that [24]. ${ }^{16}$ The price we pay is that not all CFT correlators can be reproduced in this way. Hence the problem of finding a better formalism to simultaneously take care of all W.I.'s remains open.

The key observation is that under certain conditions a derivative of a primary operator is also a primary. Thus for a completely symmetric traceless tensor with $\ell$ indexes its divergence is a primary when the dimension saturates the unitary bound $\Delta=\mathrm{d}+\ell-2$. Similarly for a completely antisymmetric tensor with $\ell$ indexes its divergence is a primary if $\Delta=\mathrm{d}-\ell$. Say, there is an antisymmetric primary $F_{\mu \nu}$ of dimension $\Delta=\mathrm{d}-2$. Its divergence $J_{\mu}=\partial_{\nu} F_{\mu \nu}$ is a primary vector field of dimension $\Delta=\mathrm{d}-1$. Besides, $J_{\mu}$ is automatically conserved!

Let's say we wish to find the general form of $\left\langle J_{\mu} \ldots\right\rangle$, where $J_{\mu}$ is a conserved current of dimension $\mathrm{d}-1$ and dots stand for some other primaries. Instead of first solving conformal W.I.'s and then imposing conservation, as we did in section 2, we can use embedding formalism to find the most general form of $\left\langle F_{\mu \nu} \ldots\right\rangle$ and then simply take a derivative. The result will automatically solve the full set of W.I.'s! It does not matter if there is such an operator $F_{\mu \nu}$ in the CFT in question, or that its dimension may violate the unitary bound. Calculating $\left\langle F_{\mu \nu} \ldots\right\rangle$ is just a mathematical trick and prior to taking the derivative it does not correspond to anything physical. Similarly, one can construct the correlators involving the stress-energy tensor starting with the correlators of a fictional primary $C_{\left(\left[\mu \mu^{\prime}\right]\left[\nu \nu^{\prime}\right]\right)}$ of dimension $\Delta=\mathrm{d}-2$ which has the symmetries of the Weyl tensor (this is discussed in more detail in [24]).

Despite simplicity and obvious advantages, unfortunately not all correlators can be obtained this way. Say, we want to calculate the following three-point function $\left\langle J_{\mu} J_{\nu} J_{\rho}\right\rangle$ (to make sure this is non-zero we can further assume the currents carry an extra color index $J_{\mu}^{a}$ which we will suppress below). One can readily find there are four linearly independent correlators of this sort, assuming $J_{\mu}$ is a primary of certain dimension (which we assumed to be $\Delta=\mathrm{d}-1$ ). After imposing conservation $\partial_{\mu} J_{\mu}=0$ only two combinations survive (we are talking about parity even correlators in a general d). Can we reproduce them using the trick with $F_{\mu \nu}$ outlined above? It is easy to show that there are four linearly-independent correlators $\left\langle F_{\mu \mu^{\prime}} F_{\nu \nu^{\prime}} F_{\rho \rho^{\prime}}\right\rangle$. But after taking the derivatives all of them become linearly dependent i.e. there is only one $\left\langle\partial_{\mu^{\prime}} F_{\mu \mu^{\prime}} \partial_{\nu^{\prime}} F_{\nu \nu^{\prime}} \partial_{\rho^{\prime}} F_{\rho \rho^{\prime}}\right\rangle$, which means the second structure of $\left\langle J_{\mu} J_{\nu} J_{\rho}\right\rangle$ can not be reproduced this way.

To make the problem even sharper let us consider the two-point function. Conformal symmetry fixes a unique $\left\langle F_{\mu \mu^{\prime}} F_{\nu \nu^{\prime}}\right\rangle$, but $\left\langle\partial_{\mu^{\prime}} F_{\mu \mu^{\prime}} \partial_{\nu^{\prime}} F_{\nu \nu^{\prime}}\right\rangle$ simply vanishes and can not reproduce the standard two-point function for conserved currents $\left\langle J_{\mu} J_{\nu}\right\rangle$. We did not find a practical way to describe those correlators that can be obtained using the $F_{\mu \nu}$ trick. Certainly for two and three-point functions this question can be answered by a direct calculation. For a four and higher-point function involving $J_{\mu}=\partial_{\nu} F_{\mu \nu}$ this is more complicated. Say, the correlator of the form $\left\langle F_{\mu \nu} \ldots\right\rangle$ has a decomposition (2.2) with the functions $f^{I}$ which we prefer to denote $f_{F}^{I_{F}}$. The corresponding correlator $\left\langle J_{\mu} \ldots\right\rangle$ will have a similar decomposition parametrized by some other functions $f^{I}$ (this time we keep

\footnotetext{
${ }^{16}$ We thank Hugh Osborn for sharing this idea with us.
} 
the original notation). Which correlators $\left\langle J_{\mu} \ldots\right\rangle$ can be obtained from $\left\langle\partial_{\nu} F_{\mu \nu} \ldots\right\rangle$ ? In terms of $f_{F}^{I_{F}}$ and $f^{I}$ this means there is a first order differential operator

$$
\hat{D}_{I_{F}}^{I}=\mathbf{A}_{I_{F}}^{I}+\mathbf{B}_{I_{F}}^{I} \frac{\partial}{\partial u}+\mathbf{C}_{I_{F}}^{I} \frac{\partial}{\partial v}
$$

which represents taking divergence of $F_{\mu \nu}$. It would be interesting to understand which $f^{I}$ 's can be obtained through $f^{I}=\hat{D}_{I_{F}}^{I} f^{I_{F}}$. In case of many $J_{\mu}$ 's such operators $\hat{D}$ should be combined leading to a differential operator of higher degree. Its nice property is that this operator will be automatically annihilated by the conservation condition (2.3) for any $f_{F}^{I_{F}}$ 's, but describing the space of possible resulting $f^{I}$ 's is not an easy task.

In case of correlators with the stress-energy tensors obtained through $C_{\left(\left[\mu \mu^{\prime}\right]\left[\nu \nu^{\prime}\right]\right)}$ the reason why not all possible structures can be obtained this way is more transparent. The resulting divergence $\partial_{\mu} T_{\mu \nu}$ is zero identically, even at the coincident points. Hence the resulting correlator $\left\langle T_{\mu \nu} \ldots\right\rangle$ would not be able to satisfy Ward Identities which include certain contact terms whenever $\partial_{\mu} T_{\mu \nu}$ inside the correlator is present. As a result the trick with $C_{\left(\left[\mu \mu^{\prime}\right]\left[\nu \nu^{\prime}\right]\right)}$ can only reproduce a part of the answer, as explained in [24].

\subsection{Solving Ward Identities in the momentum space}

In section 2 we saw that imposing conservation in the coordinate space after taking care of conformal symmetry led to a complicated problem. What if we invert the order and take care of conservation first and worry about conformal symmetry later? This could be naturally done in the momentum space: conservation of an operator $\partial_{\mu} \mathcal{O}_{\mu \ldots}=0$ would imply a linear constraint $p^{\mu} \mathcal{P}_{\mu \ldots}=0$ for the correlation function $\left\langle\mathcal{O}_{\mu \ldots}(p) \ldots\right\rangle=\mathcal{P}_{\mu \ldots}$. Such linear constraints can be easily solved explicitly. ${ }^{17}$

We were a little bit hasty to declare that we would need to solve the homogeneous constraints $p^{\mu} \mathcal{P}_{\mu \ldots}=0$. The Ward Identities responsible for the conservation of $\mathcal{O}_{\mu \ldots}$ equate the correlator $\left\langle\partial_{\mu} \mathcal{O}_{\mu \ldots} \ldots\right\rangle$ not to zero, but to a contact term. Upon taking the Fourier transform the contact term turns into a polynomial in one (or more) of the external momenta (for a simple derivation of Ward Identities in the momentum space see e.g. [29]). Thus the conservation constraint is taking the form of a system of non-homogeneous linear equations with the known right-hand-side. To illustrate this we turn to the example of the $n$-point function of the conserved currents

$$
\left\langle J_{\mu_{1}}\left(p_{1}\right) \ldots J_{\mu_{n}}\left(p_{n}\right)\right\rangle=\mathcal{P}_{\mu_{1} \ldots \mu_{n}}\left(p_{i}\right) .
$$

The conservation constraints then take the form on $n$ equations $p_{i}^{\mu_{i}} \mathcal{P}_{\ldots \mu_{i} \ldots}=\mathbf{P}_{\ldots \hat{\mu}_{i} \ldots}^{i}$ (hat means a skipped index). The right-hand-side $\mathbf{P}^{i}$ is some known combination of the $(n-1)$ point functions. Usually one can find a particular solution of this system explicitly (for example this was done in [28]) or at least this can be done in principle. The main challenge is to find a special homogeneous solution such that the full answer satisfies the conformal

\footnotetext{
${ }^{17}$ We will see below that in general solving W.I.'s in the momentum space is more challenging than in the coordinate one. Still it has some advantages. This calculation was done for the three-point function of the stress-energy tensors in $\mathrm{d}=3$ in [25], of scalars in [26, 27], and more generally of scalars, currents and stress-energy tensors in [28].
} 
Ward Identities. To this end one can write the most general solution of the conservation constraints

$$
\left\langle J_{\mu_{1}}\left(p_{1}\right) \ldots J_{\mu_{n}}\left(p_{n}\right)\right\rangle=\mathbb{P}_{\mu_{1} \ldots \mu_{n}}^{\text {particular }}\left(p_{i}\right)+\sum_{I} f^{I}\left(p_{i} \cdot p_{j}\right) \mathbb{P}_{I \mu_{1} \ldots \mu_{n}}
$$

Here $\mathbb{P}_{I}\left(p_{i}\right)$ is the basis in the space of completely transverse Lorentz-invariant tensors $p_{i}^{\mu_{i}} \mathbb{P}_{\ldots \mu_{i} \ldots}=0$ made of external momenta $p_{i}$ and functions $f_{I}$ depend on all possible Lorentz invariants $p_{i} \cdot p_{j}$.

We deliberately used the same notation for the functions $f^{I}$ in (4.3) to make it look similar to (2.2), although at this point there is not much in common. Indeed $f^{I}$ 's from (2.2) depend on $n(n-3) / 2$ conformal cross ratios and $f^{I}$ 's from (4.3) depend on $n(n-1) / 2$ Lorentz invariants. Next, the tensor structures $\mathbb{Q}_{I}$ 's live in the coordinate space and transform covariantly under conformal transformations, while the tensor structures $\mathbb{P}_{I}$ 's live in the momentum space and are transverse. Yet, quite unexpectedly the space of $\mathbb{Q}_{I}$ 's is isomorphic to the space of $\mathbb{P}_{I}$ 's! Without transversality the tensor structures $\mathbb{P}$ 's are just the general Lorentz-covariant tensor structures $\mathbb{T}_{\mathbb{I}}$ made of $n-1$ external momenta $p_{i}^{\mu}$ (here we take into account momentum conservation $\sum^{n} p_{i}=0$ ) and the flat space metric (Kronecker delta-symbol) $\delta_{\mu \nu}$. As everywhere else in the paper, the $\epsilon$-tensors are excluded because of parity. The tensors $\mathbb{T}_{\mu_{1} \ldots \mu_{n}}$ can be rewritten as a function $T\left(z_{i}\right)=\mathbb{T}_{\mu_{1} \ldots \mu_{n}} z_{1}^{\mu_{1}} \ldots z_{n}^{\mu_{n}}$ as is done in [6]. All such functions can be built of $H_{(i j)}=z_{i} \cdot z_{j}$ and $V_{i[j k]}=z_{i} \cdot\left(p_{j}-p_{k}\right)$. This is already very similar to the makeup of $\mathbb{Q}$ 's in the embedding formalism or scattering amplitudes, but at this point there is no constraint that all three indexes $i, j, k$ in $V_{i[j k]}$ must be distinct. Now, we would like to impose transversality. This can be done by multiplying each index by a projector, namely $i$-th index is contracted with a projector $\Pi_{i}^{\mu_{i} \tilde{\mu}_{i}}=\delta^{\mu_{i} \tilde{\mu}_{i}}-p_{i}^{\mu_{i}} p_{i}^{\tilde{\mu}_{i}} / p_{i}^{2}$ (the same method was also used in [28])

$$
\mathbb{P}_{\mu_{1} \ldots \mu_{n}}=\prod_{i=1}^{n} \Pi_{i \mu_{i}}^{\tilde{\mu}_{i}} \mathbb{T}_{\tilde{\mu}_{1} \ldots \tilde{\mu}_{n}} .
$$

Clearly, such projectors will annihilate all tensors which include $z_{i} \cdot p_{i}$ and therefore the space of linearly independent $V_{i[j k]}$ 's should include only those with $i \neq j \neq k$. Besides, $V_{i[j k]}$ 's trivially satisfy

$$
V_{i[j k]}+V_{i[k l]}+V_{i[l j]}=0,
$$

which precisely coincides with the constraint satisfied by $V_{i[j k]}$ 's of the embedding formalism, after a trivial redefinition of $V_{i[j k]}$ 's. Thus, we have established an isomorphism between the space of $\mathbb{P}$ 's and $\mathbb{Q}$ 's (or scattering amplitudes of massive particles).

The same logic continue to work if the correlator (4.2) also includes stress-energy tensors or conserved operators of higher spin. For example in case of stress-energy tensor (a conserved traceless symmetric tensor with two indexes) the tensor structures $\mathbb{P}_{I\left(\mu_{1} \nu_{1}\right)\left(\mu_{2} \nu_{2}\right) \ldots}$ are not only transverse but also traceless. The corresponding projector then is (see also [28])

$$
\Pi_{\mu \nu \mu^{\prime} \nu^{\prime}}=\Pi_{\mu \mu^{\prime}} \Pi_{\nu \nu^{\prime}}-\frac{1}{\mathrm{~d}-1} \Pi_{\mu \nu} \Pi_{\mu^{\prime} \nu^{\prime}}
$$


where $\Pi_{\mu \nu}$ is defined above. Clearly this projector also annihilates all $V_{i[j k]}$ unless $i \neq j \neq k$, hence establishing isomorphism between $\mathbb{P}$ 's and $\mathbb{Q}$ 's.

We have to note that the argument above is not completely rigorous unless $d$ is large enough such that the dimension of space does not affect the total number of linearly independent tensor structures $\mathbb{P}$ 's and $\mathbb{Q}$ 's. But for small $d$ there could be degenerate tensors (of the sort discussed in appendix A) when some combinations of $\mathbb{P}$ 's are zero while their counterparts made of $\mathbb{Q}$ 's are non-trivial (or vice versa). This possible complication can be avoided if we compare $\mathbb{P}$ 's in the d-dimensional space with the scattering amplitudes of massive particles $\mathbb{A}$ 's in the same space. The scattering amplitudes are equivalent classes

$$
\mathbb{A}_{\ldots \mu \ldots} \sim \mathbb{A}_{\ldots \mu \ldots}+p_{\mu}^{i}(\ldots)
$$

in the space of covariant tensors made of $(n-1)$ external momenta $p_{i}$. The tensors $\mathbb{P}$ 's span the linear subspace defined through

$$
p_{i}^{\mu} \mathbb{P}_{\ldots \mu \ldots}=0
$$

in the same space of of covariant tensors made of $(n-1)$ external momenta $p_{i}$ and $\delta_{\mu \nu}$ 's (this is the space spanned by $\mathbb{T}$ 's). The isomorphism between the space of the equivalence classes (4.7) and the linear subspace (4.8) is established with help of the orthogonal projector $\left(\Pi_{\mu \nu}\right.$ or (4.6)) which maps $\mathbb{A}$ 's into $\mathbb{P}$ 's. The opposite map is trivial. This method works in any $d$ as it takes care of the null tensors: a null tensor $\mathbb{A}$ is mapped into a null $\mathbb{P}$. Since scattering amplitudes in d dimensions in one-to-one correspondence with the conformal structures in $d-1$ we arrive at the following result: the space of $\mathbb{Q}$ 's in $d$ dimensions is isomorphic to the space of $\mathbb{P}$ 's in $d+1$.

Now let us return to (4.3) and discuss the conditions on functions $f^{I}$ such that (4.3) is conformal. These are the Ward Identities imposing the covariance under dilatation and special conformal transformations. The former is easy to satisfy as it simply requires $f^{I}$ to be homogeneous functions of certain degree in momenta (it is possible to choose a basis $\mathbb{P}_{I}$ such that each element has a definite dimension). The main complexity comes from the special conformal transformations which give rise to a system of second order differential equations. Thus, solving W.I.'s in the momentum space is significantly more involved than in the coordinate space: while the number of unknown functions in both cases $f^{I}$ is the same (in the momentum space it could be slightly smaller for small $\mathrm{d}$ ), in the momentum space these functions depend on more variables $(n(n-1) / 2$ vs. $n(n-3) / 2)$ and satisfy a system of second (rather than first) order PDEs. For example, the problem of finding the three-point function of the stress-energy tensors in the coordinate space is reduced to a simple linear algebra problem (finding a kernel of $21 \times 11$ matrix). In the momentum space the same problem requires solving a bunch of second order PDEs to determine eleven functions of three variables $f^{I}\left(p_{1}^{2}, p_{2}^{2}, p_{3}^{2}\right)$. This was only done recently in [28].

The observed connection between $\mathbb{Q}$ 's and $\mathbb{P}^{\prime}$ s suggest that solving W.I.'s in the coordinate and momentum spaces bear in common much more than was realized before. We schematically illustrate this idea in figure 2. In both coordinate and momentum space one starts by imposing Poincaré invariance i.e. representing the correlator of interest $\mathcal{P}_{\mu \ldots} \equiv\left\langle\mathcal{O}_{\mu \ldots} \ldots\right\rangle$ as a sum of all possible Lorentz-covariant tensors $\mathbb{T}_{\mathbb{I}}$ 's made of 


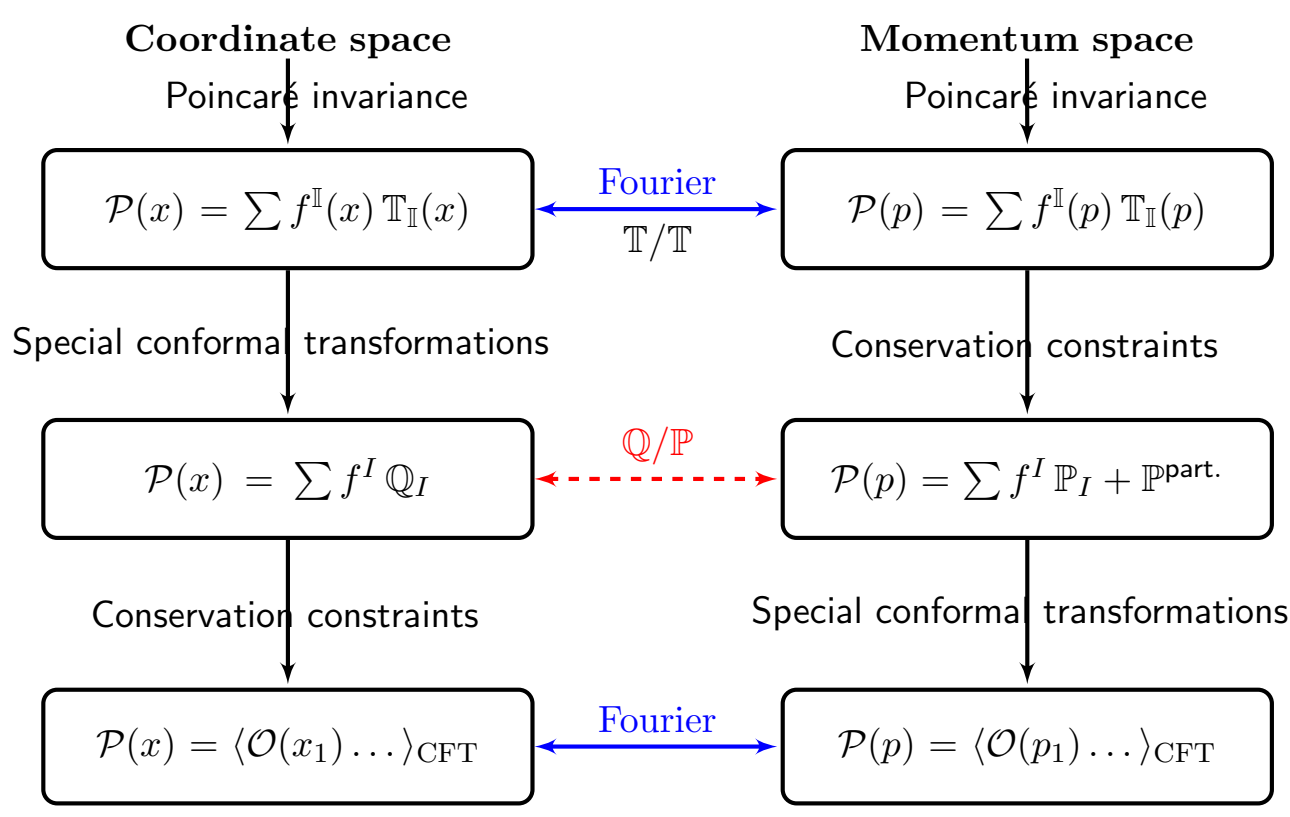

Figure 2: Parallel between solving W.I.'s in the coordinate and momentum space.

$(n-1)$ linearly independent differences $x_{i}-x_{n}$ (or $(n-1)$ linearly independent momenta $\left.p_{i}\right)$ and Kronecker delta-symbols. The functions $f^{\mathbb{I}}$ 's multiplying $\mathbb{T}$ 's depend on all Lorentzinvariant combinations of $x_{i}-x_{n}$ or $p_{i}$. Thus, at this point coordinate and momentum space representations are isomorphic. Another way to establish this isomorphism is through the Fourier transform.

The final results in the coordinate and momentum spaces, after all W.I.'s are imposed, are obviously related by the Fourier transform as well. What is interesting the intermediate results happens to be related as well. Namely the solution to the special conformal transformations constraints in the coordinate space is related to the solution of the conservation constraints in the momentum space. Strictly speaking this relation (the isomorphism between $\mathbb{Q}$ 's and $\mathbb{P}$ 's) connects the $d+1$-dimensional coordinate space with the d-dimensional momentum space, but this difference is unimportant for a sufficiently large $\mathrm{d}$ (for concrete values see appendix A). This relation strongly suggests imposing the remaining constraints, covariance under conformal transformations in the momentum space and conservation constraints in the coordinate space, should go in parallel. It is given that the conformal constraints in the momentum space are more comprehensive, as the corresponding functions $f^{I}$ depend on more variables than their counterparts in the coordinate space. But it should be possible to split the conformal constraints into two groups, such that the first group would reduce the remaining degrees of freedom in $f^{I}$ to their coordinate space counterpart, while the second group would essentially be equivalent to the analog of (2.3). It would be particularly interesting to try this logic with the three point functions of conserved currents or stress-energy tensors and explicitly isolate the group of conformal constraints which would be equivalent to the linear algebra constraints imposing conservation in the coordinate space. 


\section{Conclusions}

In this paper we have calculated the number of functional degrees of freedom surviving after imposing the full set of Ward Identities on a four-point function of stress-energy tensors or conserved currents in a d-dimensional conformal field theory. The results are presented in table 1 . These numbers precisely match the number of functional degrees of freedom governing the most general scattering amplitude of four gravitons or gauge bosons in $d+1$ dimensions. Thus our findings support the conjecture that the CFT correlators of primary operators in d dimensions are one-to-one related to the scattering amplitudes of "dual" particles in the $\mathrm{d}+1$ dimensional space. Quite remarkably this relation connects the linear algebra problem of scattering amplitudes with the differential equations problem of CFT correlators. Hence, we conjecture existence of a new formalism for the CFT correlators which would "take care" of all Ward Identities reducing them to a number of linear algebra constraints.

The number of kinematically unrestricted functions governing the 4 pt functions of the stress-energy tensors or conserved current is relatively small, much smaller than the full number of functions before the conservation of operators is taken into account. Therefore we expect that formulating and solving the conformal bootstrap constraints in term of only unrestricted degrees of freedom will have significant advantages over the naive approach which would involve many redundancies. We outlined a way to formulate the conformal bootstrap constraints in terms of only unrestricted degreed of freedom in (2.19), (2.20).

Eventually, we observed an interesting parallel between solving the full set of Ward Identities in the momentum and coordinate spaces. These findings are illustrated in figure 2.

\section{Acknowledgments}

I would like to thank Daniele Dorigoni, Simone Giombi, Sungjay Lee, Juan Maldacena, Hugh Osborn, Miguel Paulos, João Penedones, Vasily Pestun, Slava Rychkov, and Alexander Zhiboedov for discussions. I would also like to thank the Aspen Center for Physics for hospitality and gratefully acknowledge support from a Starting Grant of the European Research Council (ERC STG grant 279617), from the Taplin Fellowship and the grant RFBR 15-01-04217 as well as NSF grant 1066293.

\section{A Degenerate tensors in various dimensions}

The main ingredient in our analysis was the linear space of all Lorentz-covariant tensor structures $\mathbb{T}_{\mathbb{I}}$ made of several vectors $p_{i}^{\mu}$ and Kronecker delta-symbols $\delta_{\mu \nu}$. Tensor structures $\mathbb{P}_{I}$ or $\mathbb{Q}_{I}$ satisfying transversality or covariance under special conformal transformations form a subspace in the linear space of all covariant tensors $\mathbb{T}$ 's. Naively the space of $\mathbb{T}$ 's does not dependent on the dimension of the space d. For example for one vector $p^{\mu}$ there are two linearly independent structures with two indexes

$$
T_{\mu \nu}^{1}(p)=p_{\mu} p_{\nu}, \quad T_{\mu \nu}^{2}(p)=\delta_{\mu \nu}
$$


in any $\mathrm{d}>1$. But this is not alway the case. Whenever there are several vectors $p_{i}^{\mu}$, $1 \leq i \leq n$, certain tensors will be degenerate (i.e. identically zero for any values of $p_{i}^{\mu}$ ) for $\mathrm{d} \leq n$. In fact degenerate tensors may appear for $\mathrm{d}$ larger than $n$. For example the following tensor is zero in $\mathrm{d}=2$

$$
\begin{aligned}
& \delta_{\mu \nu} p_{\tilde{\mu}} p_{\tilde{\nu}}+p_{\mu} p_{\nu} \delta_{\tilde{\mu} \tilde{\nu}-} \text { (A.2) } \\
&\left(p_{\mu} \delta_{\nu \tilde{\mu}} p_{\tilde{\nu}}+p_{\nu} \delta_{\mu \tilde{\mu}} p_{\tilde{\nu}}+p_{\mu} \delta_{\nu \tilde{\nu}} p_{\tilde{\mu}}+p_{\nu} \delta_{\mu \tilde{\nu}} p_{\tilde{\mu}}\right) / 2+ \\
& p^{2}\left(\delta_{\mu \tilde{\mu}} \delta_{\nu \tilde{\nu}}+\delta_{\mu \tilde{\nu}} \delta_{\nu \tilde{\mu}}-2 \delta_{\mu \nu} \delta_{\tilde{\mu} \tilde{\nu}}\right) / 2
\end{aligned}
$$

This means transverse tensors $\mathbb{P}_{(\mu \nu)(\tilde{\mu} \tilde{\nu})}(p)$ or conformal tensors $\mathbb{Q}_{(\mu \nu)(\tilde{\mu} \tilde{\nu})}(p)$ in two dimensions might be degenerate as well (a reader should not be confused by our notations $\mathbb{Q}(p)$ because $p$ is an abstract vector in $\mathbb{R}^{\mathrm{d}}$, not a momentum). We do not know an analytic method to find for which $d$ the degenerate tensors would be present. Therefore we approached this problem empirically and using computer algebra we calculated the scalar product matrix by contracting all Lorentz indexes

$$
g_{\mathbb{I}}(p)=\mathbb{T}_{\mathbb{I} \mu \ldots} \mathbb{T}_{\mathbb{J} \mu \ldots}
$$

Thus, we found the scalar product matrix for the tensors $\mathbb{T}_{\mathbb{I} \mu \nu \rho}\left(p_{1}, p_{2}\right)$ the resulting scalar product is non-degenerate for all $\mathrm{d} \geq 3$. Hence all $14 \mathbb{T}_{\mathbb{I} \mu \nu \rho}\left(p_{1}, p_{2}\right)$ are linearly independent and correspondingly all $4 \mathbb{P}_{I \mu \nu \rho}\left(p_{1}, p_{2}\right)$ and $4 \mathbb{Q}_{I \mu \nu \rho}\left(p_{1}, p_{2}\right)$ are non-degenerate. Similarly we analyzed the scalar product for 5 tensors $\mathbb{T}_{\tilde{\mathbb{I}} \mu \nu}\left(p_{1}, p_{2}\right)$ which also turns out to be nondegenerate for $\mathrm{d} \geq 3$ implying linear independence of two $\tilde{\mathbb{Q}}_{\tilde{I}}{ }_{\mu \nu}$. Hence one does not have to worry about degenerate tensors while solving Ward Identities for $\left\langle J_{\mu} J_{\nu} J_{\rho}\right\rangle$ neither in momentum nor in coordinate space.

Similarly we analyzed $\mathbb{T}_{\mathbb{I} \mu \nu \rho \sigma}\left(p_{1}, p_{2}, p_{3}\right)$ and $\mathbb{T}_{\tilde{\mathbb{I}} \mu \nu \rho}\left(p_{1}, p_{2}, p_{3}\right)$ "responsible" for the $4 \mathrm{pt}$ function of currents $\left\langle J_{\mu} J_{\nu} J_{\rho} J_{\sigma}\right\rangle$. Here $\mathbb{I}$ runs up to 138 , but in $\mathrm{d}=3$ only 81 and in $\mathrm{d}=4$ only 136 are linearly independent. For $\mathrm{d} \geq 5$ there are no degeneracies. Therefore all 43 transverse tensors $\mathbb{P}_{I, \mu \nu \rho \sigma}\left(p_{1}, p_{2}, p_{3}\right)$ in $\mathrm{d} \geq 5$ are distinct, while there are only 41 of those in $\mathrm{d}=4$ and 14 in $\mathrm{d}=3$. Using the isomorphism between $\mathbb{P}$ 's in $\mathrm{d}+1$ and $\mathbb{Q}$ 's in $\mathrm{d}$ dimensions we conclude that all 43 conformal structures $\mathbb{Q}_{I, \mu \nu \rho \sigma}$ are linearly independent when $\mathrm{d} \geq 4$, and there are two degenerate structures in $\mathrm{d}=3$. These two degenerate structures are responsible for the difference between the number of unrestricted functions governing $\left\langle J_{\mu} J_{\nu} J_{\rho} J_{\sigma}\right\rangle$ in $\mathrm{d}=3$ and all other dimensions $\mathrm{d} \geq 4$ (see section 2.6). It is important to note that all $14 \tilde{\mathbb{Q}}_{\tilde{I} \mu \nu \rho}$ in $\mathrm{d} \geq 3$ are independent and therefore the analysis of section 2 in coordinate space is valid. At the same time not all $36 \tilde{\mathbb{T}}_{\tilde{\mathbb{I}} \mu \nu \rho}\left(p_{1}, p_{2}, p_{3}\right)$ are independent in $\mathrm{d}=3$, in fact there are 9 degenerate tensors of this kind. That is why solving Ward Identities for $\left\langle J_{\mu} J_{\nu} J_{\rho} J_{\sigma}\right\rangle$ in $\mathrm{d}=3$ in the momentum space would require extra care: the r.h.s. of the conservation constraint $\mathbf{P}^{i}$ introduced the section 4.2 may include extra terms which is just zero in disguise.

Before we turn to discussing the tensor structures relevant for correlators of the stressenergy tensors let us explain the origins of (A.2). Let's introduce an auxiliary metric $g_{\mu \nu}(x)$ on an asymptotically flat $\mathbb{R}^{2}$ space. The functional $W\left[g_{\mu \nu}\right]=\int \sqrt{g} R$ in $\mathrm{d}=2$ is trivial 
- it calculates Euler characteristic which is a topological quantity and hence does not depend on $g_{\mu \nu}(x)$. Thus, the first variational derivative $\delta W / \delta g^{\mu \nu}(x)$ calculated in a flat space $g_{\mu \nu}=\delta_{\mu \nu}$ is just zero. Yet the second derivative

$$
\left.\frac{\delta^{2} W}{\delta g^{\mu \nu}(x) \delta g^{\tilde{\mu} \tilde{\nu}}(y)}\right|_{g_{\mu \nu}=\delta_{\mu \nu}}
$$

will give a non-trivial expression which is zero in disguise. Upon the Fourier transform with respect to $x-y$ one obtains (A.2) which explains why it is degenerate in $\mathrm{d}=2$. The Euler characteristic exists in any even-dimensional space $\mathrm{d}=2 \mathrm{~m}$. Written in terms of local metric it is proportional to $m$-th power of Riemann curvature. Therefore first $m$ variational derivatives with respect to metric will vanish identically, while the $m+1$ th (and all higher derivatives) upon the Fourier transform would lead to a degenerate tensor in a $\mathrm{d}=2 \mathrm{~m}$-dimensional space. We observed that it would be the only degenerate tensor $\mathbb{T}_{\left(\mu_{1} \nu_{1}\right) \ldots\left(\mu_{\mathrm{m}+1} \nu_{\mathrm{m}+1}\right)}\left(p_{1}, \ldots, p_{\mathrm{m}}\right)$ with $\mathrm{m}+1$ symmetric pairs of indexes depending on $\mathrm{m}$ independent vectors in $\mathbb{R}^{2 \mathrm{~m}}$. Moreover there would be no degenerate tensors of this kind in $\mathrm{d}>2 \mathrm{~m}$ and several (or many) in $\mathrm{d}<2 \mathrm{~m}$. Furthermore there are no degenerate tensors with one index less $\mathbb{T}_{\mu_{1}\left(\mu_{2} \nu_{2}\right) \ldots\left(\mu_{\mathrm{m}+1} \nu_{\mathrm{m}+1}\right)}\left(p_{1}, \ldots, p_{\mathrm{m}}\right)$ in $\mathrm{d} \geq 2 \mathrm{~m}$ and several (or many) $\mathrm{d}<2 \mathrm{~m}$.

This simple observation can help up understand when one has to worry about degenerate tensor structures while dealing with the $n$-point function of the stress-energy tensors. Let us start with the $2 \mathrm{pt}$ function. The corresponding general Lorentz-covariant tensors $\mathbb{T}_{\mathbb{I}(\mu \nu)(\tilde{\mu} \tilde{\nu})}(p)$ depend on one vector $p^{\mu}$ and are symmetric with respect to $\mu \leftrightarrow \nu$ and $\tilde{\mu} \leftrightarrow \tilde{\nu}$. We do not require $\mathbb{T}_{\mathbb{I}}$ 's to be traceless. There are 6 of those overall and as we discussed above exactly one becomes degenerate in $\mathrm{d}=2$. We have already mentioned that all tensor structures $\tilde{\mathbb{T}}_{\tilde{\mathbb{I}} \mu(\tilde{\mu} \tilde{\nu})}(p)$ are non-degenerate in $\mathrm{d} \geq 2$ (and so obviously would be any tensor with less number of indexes). Therefore the unique zero structure in $\mathrm{d}=2$ is traceless and transverse i.e. it is one of those which we called $\mathbb{P}_{I}$ above. In fact there is just one traceless transverse tensor $\mathbb{P}_{(\mu \nu)(\tilde{\mu} \tilde{\nu})}(p)$ in any $\mathrm{d}$ and the fact that it is degenerate in $\mathrm{d}=2$ is directly responsible for the conformal anomaly: as soon as $T_{\mu \nu}$ is conserved the $2 \mathrm{pt}$ function $\left\langle T_{\mu \mu}(p) T_{\nu \nu}(-p)\right\rangle$ can not be zero. Since $\mathbb{Q}$ 's in d dimensions are isomorphic to $\mathbb{P}$ 's in $\mathrm{d}+1$, nothing pathological happens with the unique conformal structure $\mathbb{Q}_{(\mu \nu)(\tilde{\mu} \tilde{\nu})}(x-y)$ in $\mathrm{d} \geq 2$. Hence the $2 \mathrm{pt}$ of the stress-energy tensors in coordinate space is uniquely fixed and is well-defined in all $\mathrm{d}$.

Similarly, there is no degenerate tensor structures with three symmetric pairs of indexes that depend on $p_{1}, p_{2}$ in $\mathrm{d}>4$ (there are 137 of them in total). In $\mathrm{d}=4$ exactly one traceless transverse tensor becomes zero. There are already 25 zero $\mathbb{T}_{\mathbb{I}}$ 's in $\mathrm{d}=3$. Therefore the number of transverse traceless $\mathbb{P}$ 's drops from 11 in $\mathrm{d}>4$ to 10 in $\mathrm{d}=4$ and 4 in $\mathrm{d}=3$. These degenerate tensor in $\mathrm{d}=3,4$ should be taken into account while solving the Ward Identities in the momentum space $[28,29]$. Eventually, the degenerate tensor $\mathbb{P}$ in $\mathrm{d}=4$ implies there is exactly one degenerate conformal structure $\mathbb{Q}$ in $d=3$. And that is why there is one less linearly independent parity-even 3pt functions of the stress-energy tensors in $\mathrm{d}=3: 2$ instead of 3 . 
Finally, let us discuss the $4 \mathrm{pt}$ function of $T_{\mu \nu}$ 's. In $\mathrm{d} \geq 6$ there is no degenerate $\mathbb{Q}$ 's but there is exactly one zero $\mathbb{Q}$ (out of 633 ) in $\mathrm{d}=5$ (which is "dual" to the unique degenerate $\mathbb{P}$ in $\mathrm{d}=6$ ). This degenerate conformal tensor is the reason why the number of unrestricted functional D.O.F. governing the $4 \mathrm{pt}$ in $\mathrm{d}=5$ is by one smaller than in $\mathrm{d}=6$ (see table 1 ).

We conclude this section with a technical note. To calculate the scalar product matrix $g_{I J}$ for the 633 conformal structures $\mathbb{Q}_{I\left(\mu_{1} \nu_{1}\right) \ldots\left(\mu_{4} \nu_{4}\right)}\left(p_{1}, p_{2}, p_{3}\right)$ discussed above is not quite trivial because it requires first calculating a larger matrix $g_{\mathbb{I} J}$ for the most general tensors $\mathbb{T}_{\mathbb{I}\left(\mu_{1} \nu_{1}\right) \ldots\left(\mu_{4} \nu_{4}\right)}\left(p_{1}, p_{2}, p_{3}\right)$. The problem is that $g_{\mathbb{I}}$ is rather large, namely $6536 \times 6536$. Thus it would be desirable to find a way to calcualte the scalar product of $\mathbb{Q}$ 's directly, without defining it in the larger space of $\mathbb{T}$ 's first. This is in fact easy to do for the tensor structures $\mathbb{Q}$ which correspond to any four-point function i.e. which depend on three vectors. The tensor structure of $\mathbb{Q}$ 's i.e. spin of corresponding operators is unimportant. Indeed, using conformal symmetry one can bring four vectors $x_{i}^{\mu}$ to a "canonical" form when the first one vanishes, the fourth is at infinity, and the third is at unite distance along some direction $\vec{e}_{1}$. The remaining freedom is the location of the second point in a plane spanned by two vectors $\vec{e}_{1}, \vec{e}_{2}: \vec{x}_{2}=a \vec{e}_{1}+b \vec{e}_{2}$. Hence the space of conformal structures $\mathbb{Q}_{I}\left(p_{1}, p_{2}, p_{3}\right)$ is isomorphic to the space of all covariant tensors which depend on two vectors $p_{1}=a \vec{e}_{1}$ and $p_{2}=a \vec{e}_{2}$. Since in case of the stress-energy tensors we require $\mathbb{Q}$ 's to be traceless, we should impose this condition on T's as well. Finally we obtain that the space of 633 conformal structures $\mathbb{Q}_{I\left(\mu_{1} \nu_{1}\right) \ldots\left(\mu_{4} \nu_{4}\right)}\left(p_{1}, p_{2}, p_{3}\right)$ is isomprohic to the space of general traceless tensors $\mathbb{T}_{\mathbb{I}\left(\mu_{1} \nu_{1}\right) \ldots\left(\mu_{4} \nu_{4}\right)}^{\text {tracl }}\left(p_{1}, p_{2}\right)$. Similarly the 43 conformal structures $\mathbb{Q}_{I \mu \nu \rho \sigma}\left(p_{1}, p_{2}, p_{3}\right)$ corresponding to the four-point function of currents are isomorphic to $\mathbb{T}_{\mathbb{I} \mu \nu \rho \sigma}\left(p_{1}, p_{2}\right)$.

\section{B Conformal block decomposition in $\mathrm{d}=3$}

In this section we will calculate the number of unrestricted functional degrees of freedom governing the four-point function of the conserved currents in $\mathrm{d}=3$ using the conformal block decomposition. ${ }^{18}$ The general idea was explained in the end of section 3 . Since in $\mathrm{d}=3$ dimensions the only possible operators are traceless symmetric tensors with $\ell$ indexes the number of unrestricted degrees of freedom governing a correlator of four conserved currents is given by $n^{2}$ where $n$ is a number of three-point functions $\left\langle J J \mathcal{O}_{\mu_{1} \ldots \mu_{\ell}}\right\rangle$. This number was found in [30] to be 4 . Hence there are $4^{2}=16$ functional degrees of freedom governing $\langle J J J J\rangle$ in $\mathrm{d}=3$. Since 2 out of 4 three-point functions $\left\langle J J \mathcal{O}_{\mu_{1} \ldots \mu_{\ell}}\right\rangle$ are parityeven and the other two are parity odd the 16 functions split into $8+8$ responsible for the parity-even and parity-odd parts of $\langle J J J J\rangle$ correspondingly. Now we would like to analyze the action of the permutation group $\mathbb{Z}_{2} \times \mathbb{Z}_{2}$ (2.6). Depending on $\ell$ the two parity-even three-point functions are both symmetric or antisymmetric under the exchange of two $J$ 's. The resulting parity-even 4 functions contribute to the completely $\mathbb{Z}_{2} \times \mathbb{Z}_{2}$-symmetric part of $\langle J J J J\rangle$. Two parity-odd three-point functions have opposite symmetry with respect to permutation of $J$ 's for the given $\ell$. Hence the corresponding 4 functions split as follows: one is completely $\mathbb{Z}_{2} \times \mathbb{Z}_{2}$-symmetric, while the other three are odd with respect to two of

\footnotetext{
${ }^{18}$ This calculation was done together with João Penedones.
} 
the three generators (2.6) and invariant with respect to the remaining one. Eventually we have 5 functions governing the parity-even $\mathbb{Z}_{2} \times \mathbb{Z}_{2}$-symmetric part of $\langle J J J J\rangle$ and three functions each governing a non-trivial representation of $\mathbb{Z}_{2} \times \mathbb{Z}_{2}$. This counting matches our findings from section 2.6 (including non-trivial representations of $\mathbb{Z}_{2} \times \mathbb{Z}_{2}$, although we did not mention them there explicitly).

Open Access. This article is distributed under the terms of the Creative Commons Attribution License (CC-BY 4.0), which permits any use, distribution and reproduction in any medium, provided the original author(s) and source are credited.

\section{References}

[1] A.M. Polyakov, Conformal symmetry of critical fluctuations, JETP Lett. 12 (1970) 381 [Pisma Zh. Eksp. Teor. Fiz. 12 (1970) 538] [INSPIRE].

[2] A.M. Polyakov, Nonhamiltonian approach to conformal quantum field theory, Zh. Eksp. Teor. Fiz. 66 (1974) 23 [INSPIRE].

[3] H. Osborn and A.C. Petkou, Implications of conformal invariance in field theories for general dimensions, Annals Phys. 231 (1994) 311 [hep-th/9307010] [INSPIRE].

[4] H. Osborn, Implications of conformal invariance for quantum field theories in $d>2$, hep-th/9312176 [INSPIRE].

[5] D.M. Hofman and J. Maldacena, Conformal collider physics: energy and charge correlations, JHEP 05 (2008) 012 [arXiv: 0803.1467] [INSPIRE].

[6] M.S. Costa, J. Penedones, D. Poland and V.S. Rychkov, Spinning Conformal Correlators, JHEP 11 (2011) 071 [arXiv: 1107.3554] [INSPIRE].

[7] S. Raju, Four Point Functions of the Stress Tensor and Conserved Currents in AdS $S_{4} / C F T_{3}$, Phys. Rev. D 85 (2012) 126008 [arXiv:1201.6452] [INSPIRE].

[8] S. Raju, New Recursion Relations and a Flat Space Limit for AdS/CFT Correlators, Phys. Rev. D 85 (2012) 126009 [arXiv:1201.6449] [INSPIRE].

[9] R. Rattazzi, V.S. Rychkov, E. Tonni and A. Vichi, Bounding scalar operator dimensions in 4D CFT, JHEP 12 (2008) 031 [arXiv:0807.0004] [INSPIRE].

[10] V.S. Rychkov and A. Vichi, Universal Constraints on Conformal Operator Dimensions, Phys. Rev. D 80 (2009) 045006 [arXiv: 0905.2211] [InSPIRE].

[11] F. Caracciolo and V.S. Rychkov, Rigorous Limits on the Interaction Strength in Quantum Field Theory, Phys. Rev. D 81 (2010) 085037 [arXiv:0912.2726] [INSPIRE].

[12] R. Rattazzi, V.S. Rychkov and A. Vichi, Bounds in $4 D$ Conformal Field Theories with Global Symmetry, J. Phys. A 44 (2011) 035402 [arXiv:1009.5985] [InSPIRE].

[13] D. Poland and D. Simmons-Duffin, Bounds on 4D Conformal and Superconformal Field Theories, JHEP 05 (2011) 017 [arXiv: 1009.2087] [INSPIRE].

[14] R. Rattazzi, V.S. Rychkov and A. Vichi, Central Charge Bounds in $4 D$ Conformal Field Theory, Phys. Rev. D 83 (2011) 046011 [arXiv: 1009.2725] [InSPIRE].

[15] D. Poland, D. Simmons-Duffin and A. Vichi, Carving Out the Space of 4 D CFTs, JHEP 05 (2012) 110 [arXiv:1109.5176] [INSPIRE]. 
[16] V.S. Rychkov, Conformal Bootstrap in Three Dimensions?, arXiv:1111.2115 [INSPIRE].

[17] S. El-Showk, M.F. Paulos, D. Poland, V.S. Rychkov, D. Simmons-Duffin and A. Vichi, Solving the 3D Ising Model with the Conformal Bootstrap, Phys. Rev. D 86 (2012) 025022 [arXiv: 1203.6064] [INSPIRE].

[18] S. El-Showk and M.F. Paulos, Bootstrapping Conformal Field Theories with the Extremal Functional Method, Phys. Rev. Lett. 111 (2013) 241601 [arXiv:1211.2810] [InSPIRE].

[19] C. Beem, L. Rastelli and B.C. van Rees, The $\mathcal{N}=4$ superconformal bootstrap, Phys. Rev. Lett. 111 (2013) 071601 [arXiv: 1304.1803] [INSPIRE].

[20] F. Kos, D. Poland and D. Simmons-Duffin, Bootstrapping the $O(N)$ vector models, JHEP 06 (2014) 091 [arXiv: 1307.6856] [INSPIRE].

[21] L.F. Alday and A. Bissi, The superconformal bootstrap for structure constants, JHEP 09 (2014) 144 [arXiv: 1310.3757] [INSPIRE].

[22] A.A. Belavin, A.M. Polyakov and A.B. Zamolodchikov, Infinite Conformal Symmetry in Two-Dimensional Quantum Field Theory, Nucl. Phys. B 241 (1984) 333 [inSPIRE].

[23] P. Di Francesco, P. Mathieu and D. Senechal, Conformal field theory, Springer, New York U.S.A. (1997), pg. 1-890.

[24] J. Erdmenger and H. Osborn, Conserved currents and the energy momentum tensor in conformally invariant theories for general dimensions, Nucl. Phys. B 483 (1997) 431 [hep-th/9605009] [INSPIRE].

[25] J.M. Maldacena and G.L. Pimentel, On graviton non-Gaussianities during inflation, JHEP 09 (2011) 045 [arXiv: 1104.2846] [INSPIRE].

[26] C. Corianò, L. Delle Rose, E. Mottola and M. Serino, Graviton Vertices and the Mapping of Anomalous Correlators to Momentum Space for a General Conformal Field Theory, JHEP 08 (2012) 147 [arXiv:1203.1339] [INSPIRE].

[27] C. Corianò, L. Delle Rose, E. Mottola and M. Serino, Solving the Conformal Constraints for Scalar Operators in Momentum Space and the Evaluation of Feynman's Master Integrals, JHEP 07 (2013) 011 [arXiv: 1304.6944] [INSPIRE].

[28] A. Bzowski, P. McFadden and K. Skenderis, Implications of conformal invariance in momentum space, JHEP 03 (2014) 111 [arXiv: 1304.7760] [INSPIRE].

[29] A. Cappelli, R. Guida and N. Magnoli, Exact consequences of the trace anomaly in four-dimensions, Nucl. Phys. B 618 (2001) 371 [hep-th/0103237] [INSPIRE].

[30] M.S. Costa, J. Penedones, D. Poland and V.S. Rychkov, Spinning Conformal Blocks, JHEP 11 (2011) 154 [arXiv:1109.6321] [INSPIRE]. 